\title{
Space Vector vs. Sinusoidal Carrier-Based Pulse Width Modulation for a Seven-Phase Voltage Source Inverter
}

\author{
Mohammed A. Al-Hitmi, Shaikh Moinoddin, Atif Iqbal, Khaliqur Rahman, and Mohammad Meraj
}

\begin{abstract}
The paper presents an extensive comparison between two pulse width modulation (PWM) control schemes, namely carrier-based and space vector based PWM for a sevenphase voltage source inverter. Sinusoidal carrier-based PWM and space vector PWM techniques are employed to control multiphase power converters supplying variable speed multiphase motor drive systems. Implicit relationships that exist between the two PWM techniques are comprehensively explored; this paper, reveal the underlying relationship between the two PWM techniques in terms of several parameters such as modulating signals and space voltage vectors, modulating signals and space voltage vector sectors, switching pattern of space vector and type of carrier. Furthermore, sharing of zero vectors among different chosen space vectors is elaborated that leads to the formulation of different types of modulation. Digital implementation of the two modulation schemes is elaborated. Simulation results are validated using experimental investigation.
\end{abstract}

Index Terms - Carrier-based pulse width modulation (PWM), multiphase motor drive system, space vector PWM, voltage source inverter.

\section{NOMENCLATURE}

$\mathrm{a}^{*}$

$d-q$

$k$

$n$

$\xi(t)$

$V_{i}$

I to XIV

M

$m$

$\mathrm{S}_{k}(k \rightarrow 1$ to 4$)$

$t_{a 1}, t_{a 2}$ and $t_{a 3}$

$t_{b 1}, t_{b 2}$ and $t_{b 3}$

$t_{\mathrm{s}}$

$\mathrm{N}$

$V_{\mathrm{DC}}$
The transposition of ' $a$ '.

The fundamental stationary reference frame.

Number of sectors of output voltage vectors.

Total number of output phases.

Duty ratio.

Output phase voltage $(i=1,2, \ldots 7$, in volt $)$.

Sector of voltage space vector.

Maximum modulation index.

Even numbered sector of voltage space vectors.

Inverter switches.

Application time of the inverter voltage space vectors (in sec).

Application time of the inverter phase opposed voltage vectors (in sec).

Switching period (in sec).

Zero sequence voltage signal.

DC voltage source of the inverter (in volt).
Manuscript received June 10, 2019. This work was supported by Qatar University, Doha, Qatar under Grant QUCG-CENG-19/20-5.

M. A. Al-Hitmi, A. Iqbal, and M. Meraj are with the Electrical Engineering Department, Qatar University, Doha, 2713, Qatar (email: m.a.alhitmi@qu.edu.qa).

S. Moinoddin and K. Rahman are with the Electrical Engineering Department, Aligarh Muslim University, Aligarh , India (email: moinuddin2006@gmail.com).

Digital Object Identifier 10.24295/CPSSTPEA.2019.00022

$$
\begin{array}{ll}
v_{\mathrm{sm}}, v_{\mathrm{m}}, v_{1} & \text { Small, medium and large length voltage vector. } \\
V_{\max }, V_{\min } & \text { Maximum and minimum voltage max, out } \\
x_{1}-y_{1} \text { and } x_{2}-\mathrm{y}_{2} & \text { The auxiliary orthogonal planes. }
\end{array}
$$

\section{INTRODUCTION}

$\mathrm{V}$ ariable speed motor drives find widespread industrial applications ranging from manufacturing sectors, process plants, oil \& gas plants and electric and hybrid transportations. Since variable speed drives are supplied by power electronic converters to obtain variable voltage and variable frequency supply, adding extra legs become a parameter of choice. This has led to the development of multiphase power conversion and control system. A large literature is available on multiphase motor drive system modeling, analysis and control. Few review papers are available to comprehend the development in the field of multiphase motor drive systems. The first review was reported in 2002 [1] and since then work on the area has been accelerated and two reviews appeared in 2007 and 2008 [2], [3], most recently new reviews are reported in 2016 [4]-[6].

Multiphase motor drive system is known to offer numerous advantages over corresponding three-phase motor drive system. The major advantages include:1) lower torque ripple amplitude, 2) higher frequency of torque ripple, 3) higher torque density due to injection of lower order harmonics that modulate the shape of the air-gap flux, 4) less noise, 5) lower per-leg converter rating for same power output 6) higher redundancy and better fault tolerance characteristics, 7) greater control flexibility and 8) possibility of independent control of more than one machine supplied by one power converter [1]-[6].

Multiphase power converters are used to feed multiphase ac machines. Power converters used in three-phase drive are extended by adding extra legs to use in multiphase drives. Traditional two-level inverters and multilevel inverters are explored for multiphase drives. Numerous PWM techniques are used to control multiphase power converters including sinusoidal carrier-based and space vector approaches [7], [8]. Application of carrier-based PWM technique in multiphase converter is straightforward; using multiphase sinusoidal modulating signals with appropriate phase shifts and comparing with triangular high frequency signal. However, space vector PWM when applied to multiphase converter is completely different from the three-phase counterpart. When applied to an $n$-phase voltage source inverter (VSI), the total number of switching states are $2 n$ [6], [7]. The space vectors are obtained 
corresponding to the available switching states and are mapped in a polygon with $2 n$ sectors each spanning $2 \pi / n$ degree. The space vectors of a three-phase VSI when transformed in $d-q$ plane, they are spread in two-dimensional plane [7]. However, the space vectors of $n$-phase VSI can be transformed into $(n-1) / 2$ orthogonal planes. The $d-q$ plane vectors when used to implement space vector PWM, produces fundamental frequency component output with sinusoidal waveforms. While other plane vectors (auxiliary planes) e.g. $x-y$ in a fivephase and $x_{1}-y_{1}, x_{2}-y_{2}$ in a seven-phase VSIs are responsible for producing low-order harmonics and as a result distortion in the output voltage waveforms. Hence the implementation of space vector PWM require careful selection of space vectors and computation of dwell time in such a way to cancel out the effect of auxiliary plane vectors [6], [7].

Space vector PWM methods are successfully realized to cancel out the effect of auxiliary plane vectors for five-phase [9]-[12], six-phase [13]-[16], seven-phase [17], [18], ninephase [19], [20], eleven-phase [21], thirteen-phase [22], and fifteen-phase [23], [24] VSIs. Sinusoidal carrier-based PWM technique is applied to control a five-phase VSI and analyzed in [25], [26]. The maximum output voltage magnitude available when using sinusoidal carrier-based PWM technique is 0.5 $V_{\mathrm{DC}}\left(V_{\mathrm{DC}}\right.$ is the DC link voltage), irrespective of the number of phases of VSIs. The lower dc bus utilization can be improved by modifying the modulating signal either by injection $n$th harmonic or by offset addition (which is in essence multiple of $n$th harmonics) [27], [28]. This causes an increase in the range of the linear modulation.

Although the approach of both the sinusoidal carrierbased PWM and space vector PWM techniques are entirely different, however, they have an underlying relationship; this relationship is elaborated in [29], [30]. The two methods are different because of the application time of zero vectors. The zero vectors are symmetrically placed in a sample time using space vector PWM contrary to sinusoidal carrier-based PWM. When the modulating signal is modified by adding $n$th harmonic, the zero vector application is symmetrically placed and the two methods yield similar results. More comprehensive relationship between the two PWM methods are illustrated for three-phase VSI in linear modulation region [31] and over modulation region [32]. The similar approach was adopted and the relationship between carrier-based PWM and space vector is established for a five-phase VSI [33]. This paper is an extension of [34], here therelationship between the two PWM schemes is established fora seven-phase VSI. Although the extension of sinusoidalcarrier-based PWM when applied to multiphase VSI is simple, while the same is not true for space vector PWM. As in the case of multiphase space vector PWM, the total number of possible space vectors increases exponentially. There is not only increment in the number of space vectors but new subspaces are also recorded. These subspaces cause the lower order harmonics if they are not eliminated and the requirement of producing sinusoidal output voltage waveform need special considerations. Hence, the analysis presented for five-phase VSI [35], [36] cannot be easily extended to the

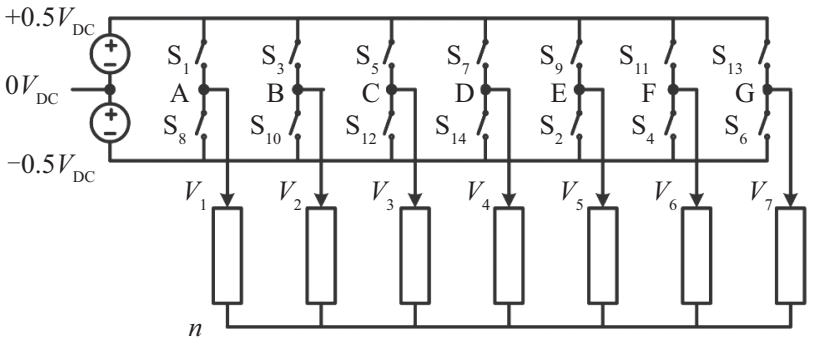

Fig. 1. Power circuit of a seven-phase voltage source inverter.

seven-phase VSI. Also the different harmonic content injection has been studied and it is experimentally validated in [37], where a third harmonic injection sinusoidal PWM method based on alternating carrier polarity is compared with the space vector PWM. The harmonic injection modulation has common mode voltage with lower frequency as compared to the space vector modulation method. Moreover, synchronization of the reference voltage and phases of injected third harmonic must be there. Whereas, in [38], the relative study on various types of carrier-based PWM techniques for multiphase induction motor drive have been presented. It is obtained that the torque pulsations in drives are intensified by the higher magnitude of space harmonics in airgap. Space harmonics can be reduced by carrier phase-shifted space vector PWM technique in which the lower centre band harmonics are cancelled and the remaining sidebands are shifted to six times of the carrier frequency. Thus, the multilevel voltage can reduce time harmonics. Better performance of the drive can be obtained by giving an optimum phase shift angle to the carriers.

\section{Seven-Phase VSI Model}

A seven-phase voltage source inverter is presented in Fig. 1. The input DC link voltage is assumed constant. The output phase voltages are represented as $v_{i}(i=1,2,3,4,5,6,7)$, in the seven-phase star-connected and the pole voltages are denoted in capital letter (A, B, C, D, E, F, G).The inverter leg/pole voltage and phase-to-neutral voltage are related as (here leg and phase voltage have the same expression [9]);

$$
v_{i}=\left(\frac{6}{7}\right) V_{j}-\left(\frac{1}{7}\right)\left(\sum_{\substack{k=A \\ k \neq j}}^{G} V_{k}\right)
$$

where, if $i=1,2 \ldots 7$, then $j=\mathrm{A}, \mathrm{B} \ldots \mathrm{G}$, respectively. It is seen from Fig. 1 that the pole/leg voltage varies from $+0.5 V_{\mathrm{DC}}$ to $-0.5 V_{\mathrm{DC}}$. The power switches can be either ON (represented by 1 ) or OFF (represented by 0 ), and hence in total $27=128$ possible states exist.

These switching states can yield 128 number of space vectors. These 128 space vectors are classified as active (126) and zero (2). Space vectors when mapped, form nine concentric tetra-decagon in the $d-q$ plane with zero space vectors at the origin [17]. In a seven-phase VSI, the space vector in the $d-q$ 
TABLE I

Lengths and Position of Phase Voltage Space Vectors [32]

\begin{tabular}{|c|c|c|c|c|}
\hline \multirow[b]{2}{*}{ Space vectors } & \multicolumn{3}{|c|}{ Set number in planes } & \multirow{2}{*}{ Space vectors lengths and position planes } \\
\hline & $d-q$ & $x_{1}-y_{1}$ & $x_{2}-y_{2}$ & \\
\hline$\underline{v}_{1 \text { phase }}$ to $\underline{v}_{14 \text { phase }}$ & 1 & 8 & 7 & $(2 / 7) V_{\mathrm{DC}} /[2 \cos (3 \pi / 7)] \exp (j k \pi / 7)$ \\
\hline$\underline{v}_{15 \text { phase }}$ to $\underline{v}_{28 \text { phase }}$ & 2 & 5 & 9 & $2(2 / 7) V_{\mathrm{DC}} \cos (\pi / 7) \exp (j k \pi / 7)$ \\
\hline$\underline{v}_{29 \text { phase }}$ to $\underline{v}_{42 \text { phase }}$ & 3 & 3 & 4 & $\sqrt{2}(2 / 7) V_{\mathrm{DC}} \exp (j 0.3052 k \pi / 7)$ \\
\hline$\underline{v}_{43 \text { phase }}$ to $\underline{v}_{56 \text { phase }}$ & 4 & 4 & 3 & $\sqrt{2}(2 / 7) V_{\mathrm{DC}} \exp (j 0.6948 k \pi / 7)$ \\
\hline$\underline{v}_{57 \text { phase }}$ to $\underline{v}_{70 \text { phase }}$ & 5 & 9 & 2 & $2(2 / 7) V_{\mathrm{DC}} \cos (2 \pi / 7) \exp (j k \pi / 7)$ \\
\hline$\underline{v}_{71 \text { phase }}$ to $\underline{v}_{84 \text { phase }}$ & 6 & 6 & 6 & $(2 / 7) V_{\mathrm{DC}} \exp (j k \pi / 7)$ \\
\hline$\underline{v}_{85 \text { phase }}$ to $\underline{v}_{98 \text { phase }}$ & 7 & 1 & 8 & $\left(2 / 7 V_{\mathrm{DC}}\right) /[2 \cos (2 \pi / 7)] \exp (j k \pi / 7)$ \\
\hline$\underline{v}_{99 \text { phase }}$ to $\underline{v}_{112 \text { phase }}$ & 8 & 7 & 1 & $\left(2 / 7 V_{\mathrm{DC}}\right) /[2 \cos (\pi / 7)] \exp (j k \pi / 7)$ \\
\hline$\underline{v}_{113 \text { phase }}$ to $\underline{v}_{126 \text { phase }}$ & 9 & 2 & 5 & $\begin{array}{l}2(2 / 7) V_{\mathrm{DC}} \cos (3 \pi / 7) \exp (j k \pi / 7) \\
\text { for } k=0,1,2 \ldots 13\end{array}$ \\
\hline$\underline{v}_{127 \text { phase }}$ to $\underline{v}_{128 \text { phase }}$ & - & - & - & 0 \\
\hline
\end{tabular}

plane is defined as:

$\underline{v}_{d q}=\frac{2}{7}\left(v_{1}+a v_{2}+a^{2} v_{3}+a^{3} v_{4}+a^{* 3} v_{5}+a^{* 2} v_{6}+a^{*} v_{7}\right)$

where $a=e^{j 2 \pi / 7}$. Using general decoupling transformation matrix voltage space vectors in the other two orthogonal planes namely $x_{1}-y_{1}$ and $x_{2}-y_{2}$ are given with,

$\underline{v}_{x_{1} y_{1}}=\frac{2}{7}\left(v_{1}+a^{2} v_{2}+a^{4} v_{3}+a^{6} v_{4}+a v_{5}+a^{3} v_{6}+a^{5} v_{7}\right)$

$\underline{v}_{x_{2} y_{2}}=\frac{2}{7}\left(v_{1}+a^{3} v_{2}+a^{6} v_{3}+a^{2} v_{4}+a^{5} v_{5}+a v_{6}+a^{4} v_{7}\right)$

The zero-sequence component is not considered since they are zero because of ungrounded neutral. The space voltage vectors are spanned over 14 sectors with each sector is spread over $25.714286^{\circ}$ ( $\pi / 7$ radians). Table I provide the detail lengths and positions of all possible 128 space vectors [32]. From Table I, it is noticed that the space vectors of $d-q$ plane are harmonics of order $14 k \pm 1, k=0,1,2,3 \ldots,(1,13,15 \ldots)$.

The space vectors of $x_{1}-y_{1}$ plane are harmonics of the order $7 k \pm 2, k=1,3,5 \ldots,(5,9,19 \ldots)$ while the space vectors of $x_{2}-y_{2}$ plane are harmonics of the order $7 k \pm 4, k=1,3,5 \ldots$, , $11,17 \ldots)$.

\section{Modelling AND Vectorial Representaion of}

\section{DifFERENT PWM SCHEMES AND RELATION BETWEen TheM}

\section{A. Space Vector Pulse Width Modulation Sinusoidal Output}

In a three-phase VSI, two neighboring space vectors are used to synthesize the reference while using space vector PWM. However, in the case of multiphase VSI, several space vectors are available in each sector. In general, implementing SVPWM require an $n-1$ number of neighboring space vectors in order to yield sinusoidal output voltage waveform [17]. This implies that six active vectors are required in each switching period in a seven-phase VSI. The time of application of space vectors should be chosen in such a way as to eliminate vectors of both $x_{1}-y_{1}$ and $x_{2}-y_{2}$ planes in order to obtain a sinusoidal outputvoltage. Thus six active vectors (In sector I, active vectors no. 1, 2, 15, 16, 71, 72 are used) and one zero vector is chosen to implement the SVPWM. The chosen vectors belong to 1st, 2 nd and 6th sets. The space vectors used in all fourteen sectors are given in [32]. Each power switch of inverter leg changes its states twice (ON to $\mathrm{OFF}$ and then $\mathrm{OFF}$ to $\mathrm{ON}$ ), hence in total twenty-eight switching takes place in one switching period.

The space vector spread in sector I is shown in Fig. 2 for $d-q, x_{1}-y_{1}$ and $x_{2}-y_{2}$ orthogonal planes. Considering equal voltsecond principle, the following relations are obtained for sector I [32]; The lengths of space vectors from Table I is obtained as:

$$
\begin{gathered}
\left|v_{1}\right|_{1-14}=\frac{V_{\mathrm{DC}}}{7 \cos \left(\frac{3 \pi}{7}\right)}\left|v_{\mathrm{m}}\right|_{15-28}=\frac{4 V_{\mathrm{DC}} \cos \left(\frac{\pi}{7}\right)}{7}\left|v_{\mathrm{sm}}\right|_{71-84}=\frac{2 V_{\mathrm{DC}}}{7} \\
k_{1}=\frac{\left|v_{\mathrm{sm}}\right|}{\left|v_{1}\right|}=0.44504 \\
k_{2}=\frac{\left|v_{\mathrm{m}}\right|}{\left|v_{1}\right|}=0.8019377 \\
k_{3}=k_{1}+k_{2}+1=2.246979
\end{gathered}
$$

From [18],

$$
\begin{aligned}
& t_{a}=\frac{\left|v_{\mathrm{s}}^{*}\right| \sin \left(k \frac{\pi}{7}-\theta\right)}{\left|v_{1}\right| \sin \left(\frac{\pi}{7}\right)} t \\
& t_{b}=\frac{\left|v_{\mathrm{s}}^{*}\right| \sin \left[\theta-(k-1) \frac{\pi}{7}\right]}{\left|v_{1}\right| \sin \left(\frac{\pi}{7}\right)}
\end{aligned}
$$

where $k=1,2, \ldots .13,14$.

Let $t_{a 3}, t_{a 2}$ and $t_{a 1}$ be the time of application for vectors numbers sets 71-84, 15-28 and 1-14, respectively. Similarly, let the times of applications $t_{b 3}, t_{b 2}$ and $t_{b 1}$ be the dwell time for another side of vectors number sets 71-84, 15-28 and $1-14$, respectively. Therefore, the time of applications of space vectors can be written in (3)-(4). The time of application of (4) completely agree with [17], [18]. The zero space vector application time is $t_{a 1}, t_{a 2}$ and $t_{a 3}$ are application times of space vectors 1,15 , and 71 , respectively and $t_{b 1}, t_{b 2}$ and $t_{b 3}$ are applications times of space vectors 2,16 and 72, respectively in sector I. Fig. 3 shows the switching sequence and decomposition of vectors in sector I.

\section{B. Carrier-Based Sinusoidal PWM (CBSPWM)}

CBSPWM techniques are simple in implementing analogically and digitally, hence, they are popular and widely used [26]. 

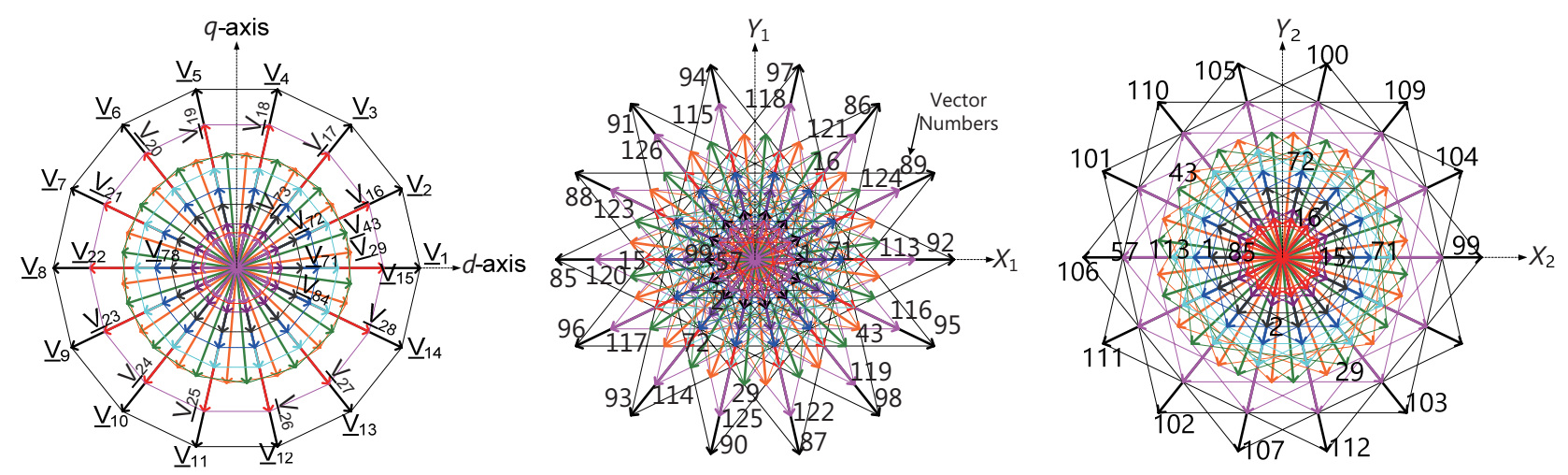

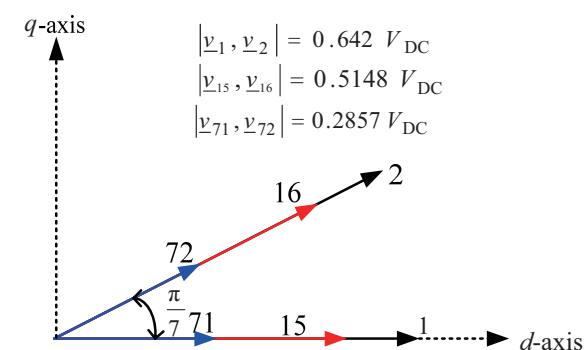

(a)

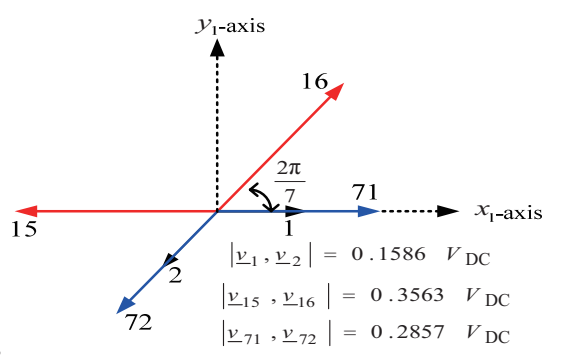

(b)

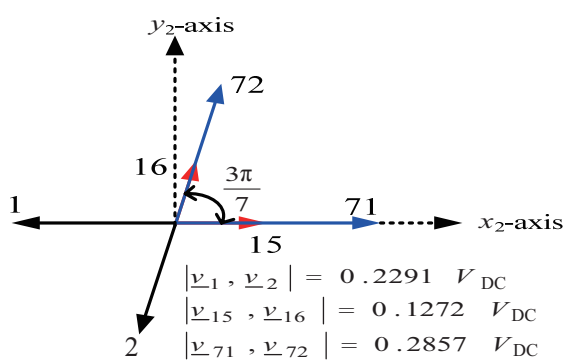

(c)

Fig. 2. Space vector disposition in sector I. (a) $d-q$ plane. (b) $x_{1}-y_{1}$ plane. (c) $x_{2}-y_{2}$ plane.

Sector I

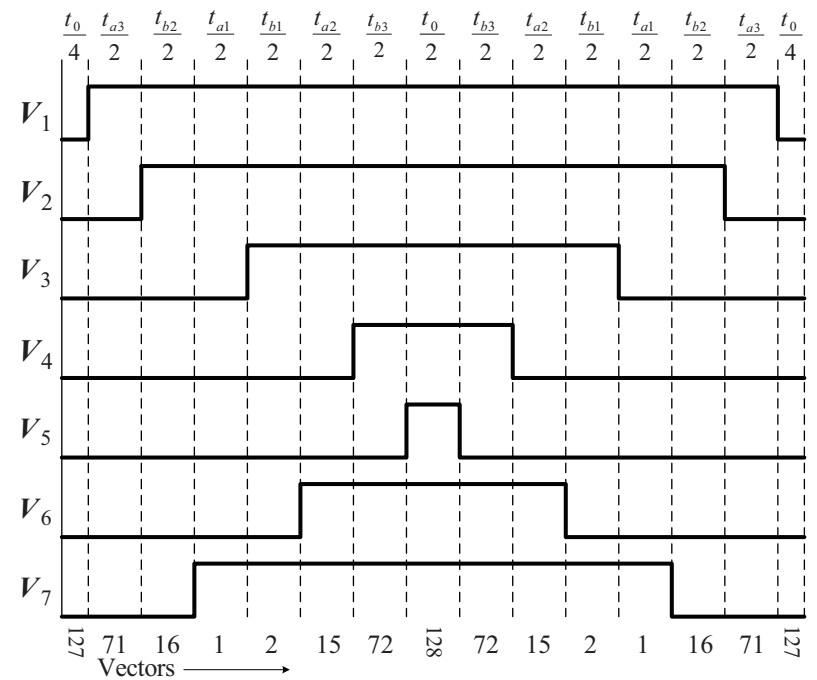

(a)

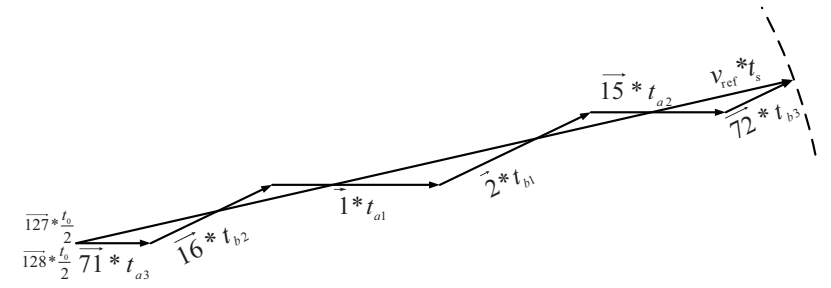

(b)

Fig. 3. (a) Switching pattern and space vector disposition for one cycle of operation. (b) Sketch of vector decomposition on volt.sec principle to eliminate/reduce harmonics.
The principle of application of CBSPWM to a multiphase VSI is the same as that for a three-phase VSI. The sinusoidal modulating signals are compared with a triangular or a saw-tooth carrier signal to generate PWM signals. The frequency of carrier signal is kept much higher than that of the modulating signals.

$$
\begin{aligned}
& t_{a 1}=\frac{\left|v_{\mathrm{s}}^{*}\right| \sin \left(k \frac{\pi}{7}-\theta\right)}{k_{3}\left|v_{1}\right| \sin \left(\frac{\pi}{7}\right)} t_{\mathrm{s}} \\
& t_{a 2}=\frac{k_{2}\left|v_{\mathrm{s}}^{*}\right| \sin \left(k \frac{\pi}{7}-\theta\right)}{k_{3}\left|v_{1}\right| \sin \left(\frac{\pi}{7}\right)} t_{\mathrm{s}} \\
& t_{a 3}=\frac{k_{1}\left|v_{\mathrm{s}}^{*}\right| \sin \left(k \frac{\pi}{7}-\theta\right)}{k_{3}\left|v_{1}\right| \sin \left(\frac{\pi}{7}\right)} t_{\mathrm{s}} \\
& t_{b 1}=\frac{\left|v_{\mathrm{s}}^{*}\right| \sin \left[\theta-(k-1) \frac{\pi}{7}\right]}{k_{3}\left|v_{1}\right| \sin \left(\frac{\pi}{7}\right)} t_{\mathrm{s}} \\
& t_{b 2}=\frac{k_{2}\left|v_{\mathrm{s}}^{*}\right| \sin \left[\theta-(k-1) \frac{\pi}{7}\right]}{k_{b 3}\left|v_{1}\right| \sin \left(\frac{\pi}{7}\right)} t_{\mathrm{s}} \\
& k_{1}\left|v_{\mathrm{s}}^{*}\right| \sin \left[\theta-(k-1) \frac{\pi}{7}\right] \\
& k_{3}\left|v_{1}\right| \sin \left(\frac{\pi}{7}\right)
\end{aligned}
$$


Substituting the values of $k_{1}, k_{2}$ and $k_{3}$ in (3) one get,

$$
\begin{aligned}
& t_{a 1}=0.445 \frac{\left|v_{\mathrm{s}}^{*}\right| \sin \left(k \frac{\pi}{7}-\theta\right)}{\left|v_{1}\right| \sin \left(\frac{\pi}{7}\right)} t_{\mathrm{s}} \\
& t_{a 2}=0.3569 \frac{\left|v_{\mathrm{s}}{ }^{*}\right| \sin \left(k \frac{\pi}{7}-\theta\right)}{\left|v_{1}\right| \sin \left(\frac{\pi}{7}\right)} t_{\mathrm{s}} \\
& t_{a 3}=0.19806 \frac{\left|v_{\mathrm{s}}\right| \sin \left(k \frac{\pi}{7}-\theta \mid\right.}{\left|v_{1}\right| \sin \left(\frac{\pi}{7}\right)} t_{\mathrm{s}} \\
& t_{b 1}=0.445 \frac{\left|v_{\mathrm{s}}{ }^{*}\right| \sin \left[\theta-(k-1) \frac{\pi}{7}\right]}{\left|v_{1}\right| \sin \left(\frac{\pi}{7}\right)} t_{\mathrm{s}} \\
& t_{b 2}=0.3569 \frac{\left|v_{\mathrm{s}}^{*}\right| \sin \left[\theta-(k-1) \frac{\pi}{7}\right]}{\left|v_{1}\right| \sin \left(\frac{\pi}{7}\right)} t_{\mathrm{s}} \\
& t_{b 3}=0.19806 \frac{\left|v_{\mathrm{s}}\right| \sin \left[\theta-(k-1) \frac{\pi}{7}\right]}{\mathrm{s}} t_{\mathrm{s}}
\end{aligned}
$$

\section{1) Sinusoidal Pulse Width Modulation (SPWM)}

The SPWM becomes simple when the zero-sequence signal is set to zero. PWM signals for all legs are as per sinusoidal fundamental signals. The highest modulation index in the linear region is unity, it is defined as:

$$
M=\frac{V_{1}}{0.5 V_{\mathrm{DC}}}
$$

where, $V_{1}$ is the fundamental output phase voltage.

\section{2) Seventh Harmonic Injection PWM-SHIPWM}

To enhance the output voltage magnitude in a carrier-based PWM, modulating signal is augmented by injecting $n$-th harmonic. This causes a reduction in peak of the modulating signal providing further room for pushing the modulation index. In general, the amount of $n$-th harmonic to be injected into the fundamental is given as [25].

$$
V_{n}= \pm V_{1} \frac{\sin (\pi / 2 n)}{n}
$$

where (6) takes the positive sign for $n=3,7,11,15 \ldots$ and negative sign for $n=5,9,13,17 \ldots$ (number of phases). Hence in a seven-phase system, the injected 7th harmonic is in phase with the fundamental. The zero-sequence signal is thus defined as:

$$
v_{n \mathrm{~N}}(t)=V_{7} \sin (7 \omega t) \frac{V_{1}}{7} \sin \left(\frac{\pi}{14}\right) \sin (7 \omega t)
$$

Using (7), the generalized resulting modulating signals have

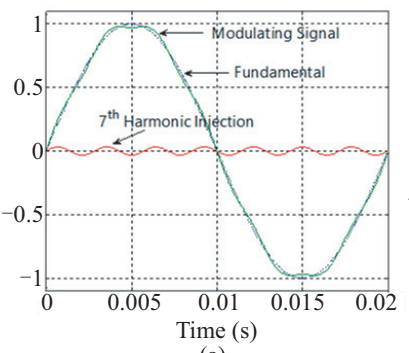

(a)

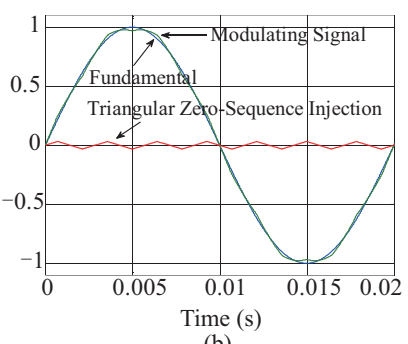

Fig. 4. Characteristics signals of carrier-based PWM. (a) With seventh harmonic injection. (b) With triangular harmonic injection.

the form

$$
V_{i}=M \cdot 0.5 V_{\mathrm{DC}}\left[\sin \left(\omega t-\frac{m \pi}{7}\right)+0.0318 \cdot \sin (7 \omega t)\right]
$$

where $i=1,2, \ldots, 7 ; m=0,2,4, \ldots, 10,12$. In the linear region, when $n$-th harmonic injected, the maximum modu-lation index $(M)$ is expressed as [25]:

$$
M=\frac{1}{\cos \left(\frac{\pi}{2 n}\right)}
$$

where $n=7$, the maximum modulation index $M_{\mathrm{SHIPWM}}=$ 1.0257. Thus there is $2.57 \%$ increase in the DC bus utilization comparing SPWM.

\section{3) Offset Addition PWM or Triangular Injection PWM (TIPWM)}

Another prevalent approach to enhance the output voltage magnitude in a carrier-based PWM scheme is by adding an offset signal into the fundamental signal. The modulating signal is modified in such a way that its peak shrinks providing an additional room for pushing the reference value. The offset signal is given as:

$$
v_{n \mathrm{~N}}=-\frac{1}{2}\left(V_{\max }+V_{\min }\right)
$$

where, $V_{\max }=\max \left(V_{1}, V_{2}, V_{3}, V_{4}, V_{5}, V_{6}, V_{7}\right)$ and $V_{\min }=$ $\min \left(V_{1}, V_{2}, V_{3}, V_{4}, V_{5}, V_{6}, V_{7}\right)$. The voltage $v_{n \mathrm{~N}}$ (the modulating signal) contains all multiple of 7 th harmonics. If the same modulating signal is used to generate the gating signals the maximum achievable modulation index would be $M_{\mathrm{TIPWM}}=$ 1.0257. To illustrate the effects of 7 th harmonic and offset signal injection the overall modulating signals are shown in Fig. 4.

\section{Relationship Between Carrier-Based and Space Vector $P W M$}

Fig. 5 shows the generated PWM switching signals when the triangular carrier signal interacts with the sinusoidal signals. It can be seen that the CBPWM (Fig. 5) and SVPWM (Fig. 6) have the similar pattern in sector I as well as in other sectors too. From the switching pattern, the relationship between space vector application time in different sectors and the modulating 
TABLE II

Relationship Between Modulating Signals and Space Vector Application Time

\begin{tabular}{|c|c|c|c|c|c|c|c|}
\hline $\begin{array}{l}\text { Signals } \\
\text { sectors }\end{array}$ & $V_{1}$ & $V_{2}$ & $V_{3}$ & $V_{4}$ & $V_{5}$ & $V_{6}$ & $V_{7}$ \\
\hline I & $\begin{array}{l}\left(V_{\mathrm{DC}} / 2 t_{\mathrm{s}}\right)\left(t_{a 1}+\right. \\
t_{b 1}+t_{a 2}+t_{b 2}+ \\
\left.t_{a 3}+t_{b 3}\right)\end{array}$ & $\begin{array}{l}\left(V_{\mathrm{DC}} / 2 t_{\mathrm{s}}\right)\left(t_{a 1}+\right. \\
t_{b 1}+t_{a 2}+t_{b 2}- \\
\left.t_{a 3}+t_{b 3}\right)\end{array}$ & $\begin{array}{l}\left(V_{\mathrm{DC}} / 2 t_{\mathrm{s}}\right)(- \\
t_{a 1}+t_{b 1}+t_{a 2}- \\
\left.t_{b 2}-t_{a 3}+t_{b 3}\right)\end{array}$ & $\begin{array}{l}\left(V_{\mathrm{DC}} / 2 t_{\mathrm{s}}\right)\left(-t_{a 1}\right. \\
-t_{b 1}-t_{a 2}-t_{b 2} \\
\left.-t_{a 3}+t_{b 3}\right)\end{array}$ & $\begin{array}{l}\left(V_{\mathrm{DC}} / 2 t_{\mathrm{s}}\right)\left(-t_{a 1}\right. \\
-t_{b 1}-t_{a 2}-t_{b 2} \\
\left.-t_{a 3}-t_{b 3}\right)\end{array}$ & $\begin{array}{l}\left(V_{\mathrm{DC}} / 2 t_{\mathrm{s}}\right)\left(-t_{a 1}\right. \\
-t_{b 1}+t_{a 2}-t_{b 2} \\
\left.-t_{a 3}+t_{b 3}\right)\end{array}$ & $\begin{array}{l}\left(V_{\mathrm{DC}} / 2 t_{\mathrm{s}}\right)\left(t_{a 1}+\right. \\
t_{b 1}+t_{a 2}-t_{b 2} \\
\left.-t_{a 3}+t_{b 3}\right)\end{array}$ \\
\hline II & $\begin{array}{l}\left(V_{\mathrm{DC}} / 2 t_{\mathrm{s}}\right)\left(t_{a 1}+\right. \\
t_{b 1}+t_{a 2}+t_{b 2}+ \\
\left.t_{a 3}-t_{b 3}\right)\end{array}$ & $\begin{aligned} & \left(V_{\mathrm{DC}} / 2 t_{\mathrm{s}}\right)\left(t_{a 1}\right. \\
+ & t_{b 1}+t_{a 2}+t_{b 2} \\
+ & \left.t_{a 3}+t_{b 3}\right)\end{aligned}$ & $\begin{aligned} & \left(V_{\mathrm{DC}} / 2 t_{\mathrm{s}}\right)\left(t_{a 1}\right. \\
+ & t_{b 1}-t_{a 2}+t_{b 2} \\
+ & \left.t_{a 3}-t_{b 3}\right)\end{aligned}$ & $\begin{aligned} & \left(V_{\mathrm{DC}} / 2 t_{\mathrm{s}}\right)\left(-t_{a 1}\right. \\
- & t_{b 1}-t_{a 2}+t_{b 2} \\
+ & \left.t_{a 3}-t_{b 3}\right)\end{aligned}$ & $\begin{array}{l}\left(V_{\mathrm{DC}} / 2 t_{\mathrm{s}}\right)\left(-t_{a 1}\right. \\
-t_{b 1}-t_{a 2}-t_{b 2} \\
\left.-t_{a 3}-t_{b 3}\right)\end{array}$ & $\begin{array}{l}\left(V_{\left.\mathrm{DC} / 2 t_{\mathrm{s}}\right)\left(-t_{a 1}\right.}\right. \\
-t_{b 1}-t_{a 2}-t_{b 2} \\
\left.+t_{a 3}-t_{b 3}\right)\end{array}$ & $\begin{array}{l}\left(V_{\mathrm{DC}} / 2 t_{\mathrm{s}}\right)\left(t_{a 1}-\right. \\
t_{b 1}-t_{a 2}+t_{b 2} \\
\left.+t_{a 3}-t_{b 3}\right)\end{array}$ \\
\hline III & $\begin{array}{l}\left(V_{\mathrm{DC}} / 2 t_{\mathrm{s}}\right)\left(t_{a 1}+\right. \\
t_{b 1}+t_{a 2}-t_{b 2}- \\
\left.t_{a 3}+t_{b 3}\right)\end{array}$ & $\begin{aligned} & \left(V_{\mathrm{DC}} / 2 t_{\mathrm{s}}\right)\left(t_{a 1}\right. \\
+ & t_{b 1}+t_{a 2}+t_{b 2} \\
+ & \left.t_{a 3}+t_{b 3}\right)\end{aligned}$ & $\begin{aligned} & \left(V_{\mathrm{DC}} / 2 t_{\mathrm{s}}\right)\left(t_{a 1}\right. \\
+ & t_{b 1}+t_{a 2}+t_{b 2} \\
- & \left.t_{a 3}+t_{b 3}\right)\end{aligned}$ & $\begin{aligned} & \left(V_{\mathrm{DC}} / 2 t_{\mathrm{s}}\right)\left(-t_{a 1}\right. \\
+ & t_{b 1}+t_{a 2}-t_{b 2} \\
- & \left.t_{a 3}+t_{b 3}\right)\end{aligned}$ & $\begin{aligned} & \left(V_{\mathrm{DC}} / 2 t_{\mathrm{s}}\right)\left(-t_{a 1}\right. \\
- & t_{b 1}-t_{a 2}-t_{b 2} \\
- & \left.t_{a 3}+t_{b 3}\right)\end{aligned}$ & $\begin{array}{l}\left(V_{\left.\mathrm{DC} / 2 t_{\mathrm{s}}\right)\left(-t_{a 1}\right.}\right. \\
-t_{b 1}-t_{a 2}-t_{b 2} \\
\left.-t_{a 3}-t_{b 3}\right)\end{array}$ & $\begin{array}{l}\left(V_{\mathrm{DC}} / 2 t_{\mathrm{s}}\right)\left(-t_{a 1}\right. \\
-t_{b 1}+t_{a 2}-t_{b 2} \\
\left.-t_{a 3}+t_{b 3}\right)\end{array}$ \\
\hline IV & $\begin{array}{l}\left(V_{\mathrm{DC}} / 2 t_{\mathrm{s}}\right)\left(t_{a 1}\right. \\
-t_{b 1}-t_{a 2}+t_{b 2} \\
\left.+t_{a 3}-t_{b 3}\right)\end{array}$ & $\begin{array}{l}\left(V_{\mathrm{DC}} / 2 t_{\mathrm{s}}\right)\left(t_{a 1}+\right. \\
t_{b 1}+t_{a 2}+t_{b 2}+ \\
\left.t_{a 3}-t_{b 3}\right)\end{array}$ & $\begin{array}{l}\left(V_{\mathrm{DC}} / 2 t_{\mathrm{s}}\right)\left(t_{a 1}+\right. \\
t_{b 1}+t_{a 2}+t_{b 2} \\
\left.+t_{a 3}+t_{b 3}\right)\end{array}$ & $\begin{array}{l}\left(V_{\mathrm{DC}} / 2 t_{\mathrm{s}}\right)\left(t_{a 1}+\right. \\
t_{b 1}-t_{a 2}+t_{b 2} \\
\left.+t_{a 3}-t_{b 3}\right)\end{array}$ & $\begin{array}{l}\left(V_{\mathrm{DC}} / 2 t_{\mathrm{s}}\right)\left(-t_{a 1}\right. \\
-t_{b 1}-t_{a 2}+t_{b 2} \\
\left.+t_{a 3}-t_{b 3}\right)\end{array}$ & $\begin{array}{l}\left(V_{\mathrm{DC}} / 2 t_{\mathrm{s}}\right)\left(-t_{a 1}\right. \\
-t_{b 1}-t_{a 2}-t_{b 2} \\
\left.-t_{a 3}-t_{b 3}\right)\end{array}$ & $\begin{array}{l}\left(V_{\mathrm{DC}} / 2 t_{\mathrm{s}}\right)\left(-t_{a 1}\right. \\
-t_{b 1}-t_{a 2}-t_{b 2} \\
\left.+t_{a 3}-t_{b 3}\right)\end{array}$ \\
\hline $\mathrm{V}$ & $\begin{array}{l}\left(V_{\mathrm{DC}} / 2 t_{\mathrm{s}}\right)\left(-t_{a 1}\right. \\
-t_{b 1}+t_{a 2}-t_{b 2} \\
\left.-t_{a 3}+t_{b 3}\right)\end{array}$ & $\begin{array}{l}\left(V_{\mathrm{DC}} / 2 t_{\mathrm{s}}\right)\left(t_{a 1}+\right. \\
t_{b 1}+t_{a 2}-t_{b 2}- \\
\left.t_{a 3}+t_{b 3}\right)\end{array}$ & $\begin{array}{l}\left(V_{\mathrm{DC}} / 2 t_{\mathrm{s}}\right)\left(t_{a 1}+\right. \\
t_{b 1}+t_{a 2}+t_{b 2} \\
\left.+t_{a 3}+t_{b 3}\right)\end{array}$ & $\begin{array}{l}\left(V_{\mathrm{DC}} / 2 t_{\mathrm{s}}\right)\left(t_{a 1}+\right. \\
t_{b 1}+t_{a 2}+t_{b 2} \\
\left.-t_{a 3}+t_{b 3}\right)\end{array}$ & $\begin{array}{l}\left(V_{\mathrm{DC}} / 2 t_{\mathrm{s}}\right)\left(-t_{a 1}\right. \\
+t_{b 1}+t_{a 2}-t_{b 2} \\
\left.-t_{a 3}+t_{b 3}\right)\end{array}$ & $\begin{array}{l}\left(V_{\mathrm{DC}} / 2 t_{\mathrm{s}}\right)\left(-t_{a 1}\right. \\
-t_{b 1}-t_{a 2}-t_{b 2} \\
\left.-t_{a 3}+t_{b 3}\right)\end{array}$ & $\begin{array}{l}\left(V_{\mathrm{DC}} / 2 t_{\mathrm{s}}\right)\left(-t_{a 1}\right. \\
-t_{b 1}-t_{a 2}-t_{b 2} \\
\left.-t_{a 3}-t_{b 3}\right)\end{array}$ \\
\hline VI & $\begin{aligned} & \left(V_{\mathrm{DC}} / 2 t_{\mathrm{s}}\right)\left(-t_{a 1}\right. \\
- & t_{b 1}-t_{a 2}-t_{b 2} \\
+ & \left.t_{a 3}-t_{b 3}\right)\end{aligned}$ & $\begin{array}{l}\left(V_{\mathrm{DC}} / 2 t_{\mathrm{s}}\right)\left(t_{a 1}-\right. \\
t_{b 1}-t_{a 2}+t_{b 2}+ \\
\left.t_{a 3}-t_{b 3}\right)\end{array}$ & $\begin{array}{l}\left(V_{\mathrm{DC}} / 2 t_{\mathrm{s}}\right)\left(t_{a 1}+\right. \\
t_{b 1}+t_{a 2}+t_{b 2} \\
\left.+t_{a 3}-t_{b 3}\right)\end{array}$ & $\begin{array}{l}\left(V_{\mathrm{DC}} / 2 t_{\mathrm{s}}\right)\left(t_{a 1}+\right. \\
t_{b 1}+t_{a 2}+t_{b 2} \\
\left.+t_{a 3}+t_{b 3}\right)\end{array}$ & $\begin{array}{l}\left(V_{\mathrm{DC}} / 2 t_{\mathrm{s}}\right)\left(t_{a 1}+\right. \\
t_{b 1}-t_{a 2}+t_{b 2} \\
\left.+t_{a 3}-t_{b 3}\right)\end{array}$ & $\begin{array}{l}\left(V_{\mathrm{DC}} / 2 t_{\mathrm{s}}\right)\left(-t_{a 1}\right. \\
-t_{b 1}-t_{a 2}+t_{b 2} \\
\left.+t_{a 3}-t_{b 3}\right)\end{array}$ & $\begin{array}{l}\left(V_{\mathrm{DC}} / 2 t_{\mathrm{s}}\right)\left(-t_{a 1}\right. \\
-t_{b 1}-t_{a 2}-t_{b 2} \\
\left.-t_{a 3}-t_{b 3}\right)\end{array}$ \\
\hline VII & $\begin{array}{l}\left(V_{\mathrm{DC}} / 2 t_{\mathrm{s}}\right)\left(-t_{a 1}\right. \\
-t_{b 1}-t_{a 2}-t_{b 2} \\
\left.-t_{a 3}-t_{b 3}\right)\end{array}$ & $\begin{array}{l}\left(V_{\mathrm{DC}} / 2 t_{\mathrm{s}}\right)\left(-t_{a 1}\right. \\
-t_{b 1}+t_{a 2}-t_{b 2} \\
\left.-t_{a 3}+t_{b 3}\right)\end{array}$ & $\begin{array}{l}\left(V_{\mathrm{DC}} / 2 t_{\mathrm{s}}\right)\left(t_{a 1}+\right. \\
t_{b 1}+t_{a 2}-t_{b 2}- \\
\left.t_{a 3}+t_{b 3}\right)\end{array}$ & $\begin{array}{l}\left(V_{\mathrm{DC}} / 2 t_{\mathrm{s}}\right)\left(t_{a 1}+\right. \\
t_{b 1}+t_{a 2}+t_{b 2} \\
\left.+t_{a 3}+t_{b 3}\right)\end{array}$ & $\begin{array}{l}\left(V_{\mathrm{DC}} / 2 t_{\mathrm{s}}\right)\left(t_{a 1}+\right. \\
t_{b 1}+t_{a 2}+t_{b 2} \\
\left.-t_{a 3}+t_{b 3}\right)\end{array}$ & $\begin{array}{l}\left(V_{\mathrm{DC}} / 2 t_{\mathrm{s}}\right)\left(-t_{a 1}\right. \\
+t_{b 1}+t_{a 2}-t_{b 2} \\
\left.-t_{a 3}+t_{b 3}\right)\end{array}$ & $\begin{array}{l}\left(V_{\mathrm{DC}} / 2 t_{\mathrm{s}}\right)\left(-t_{a 1}\right. \\
-t_{b 1}-t_{a 2}-t_{b 2} \\
\left.-t_{a 3}+t_{b 3}\right)\end{array}$ \\
\hline
\end{tabular}

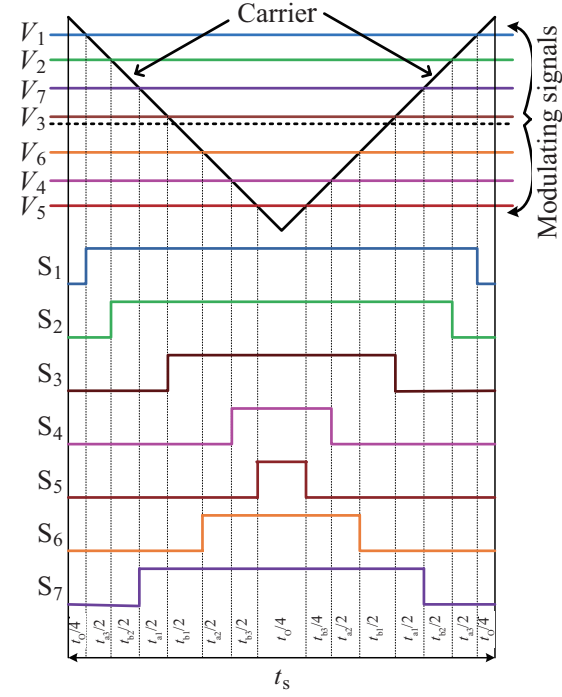

Fig. 5. SVPWM and CBPWM signals in sector I.

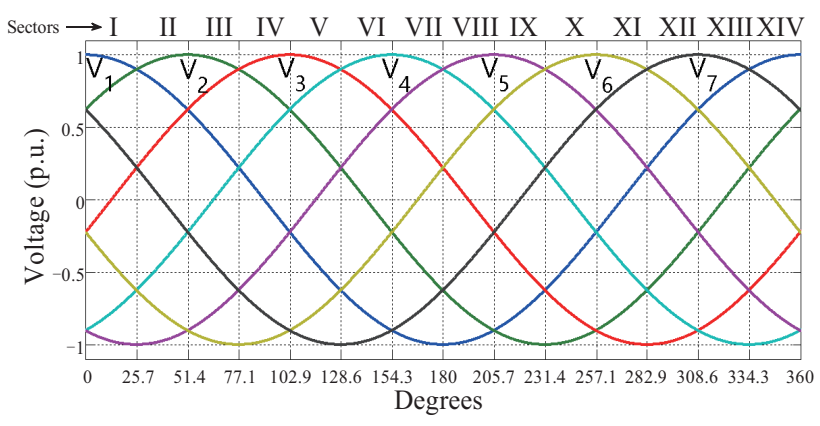

Fig. 6. Relationship between space vector sectors and fundamental signals. signals can be found. They are tabulated in Table II. The same relationship exists for the sectors from VIII to XIV but with opposite polarity to that of sectors I to VII, respectively. The zero-sequence signal can then be found as given in (11).

$$
v_{n \mathrm{~N}}=\frac{1}{7}\left(V_{1}+V_{2}+V_{3}+V_{4}+V_{5}+V_{6}+V_{7}\right)
$$

The relationships can be obtained between the zero-sequence signal and space vector application time using (11) and the switching pattern for all fourteen sectors. They are listed in Table III for sectors I to VII. Again, the same relationship exists for the sectors from VIII to XIV but with opposite polarity to that of sectors I to VII, respectively. The general expression for zero-sequence signal in even and odd sectors can be expressed as in (12) and (13), respectively.

$$
\begin{aligned}
& v_{n \mathrm{~N}}=\frac{V_{\mathrm{DC}}}{2 t_{\mathrm{s}}}\left(t_{a 1}-t_{b 1}-3 t_{a 2}+3 t_{b 2}+5 t_{a 3}-5 t_{b 3}-7 t_{127}+7 t_{128}\right) \\
& v_{n \mathrm{~N}}=\frac{V_{\mathrm{DC}}}{2 t_{\mathrm{s}}}\left(-t_{a 1}+t_{b 1}+3 t_{a 2}-3 t_{b 2}-5 t_{a 3}+5 t_{b 3}-7 t_{127}+7 t_{128}\right)
\end{aligned}
$$

\section{Relationship Between Space Vectors and Line-to-Line Voltages}

In a seven-phase system three systems of line-to-line voltages namely adjacent, 1 st non-adjacent, and 2nd non-adjacent, exist. The relationship between line-to-line voltage and space vectors can be obtained using equations of Table II and equation (14), given in Table IV for sectors I and II;

$$
v_{i j} t_{\mathrm{s}}=\left(v_{i}-v_{j}\right) t_{\mathrm{s}}=\frac{V_{\mathrm{DC}}}{2}\left(v_{i}^{*}-v_{j}^{*}\right) t_{\mathrm{s}}
$$

where $i, j=1,2,3,4,5,6,7$ and $i \neq j$. Once again, the same 
TABLE III

Zero-Sequence Signals in Different Sectors

\begin{tabular}{lll}
\hline \hline Sector No. & \multicolumn{1}{c}{ Switching period } & Zero-sequence signal \\
\hline I & $t_{a 1}+t_{b 1}+t_{a 2}+t_{b 2}+t_{a 3}+t_{b 3}-t_{127}+t_{128}$ & $v_{n \mathrm{~N}}=\frac{V_{\mathrm{DC}}}{2 t_{\mathrm{s}}}\left(-t_{a 1}+t_{b 1}+3 t_{a 2}-3 t_{b 2}-5 t_{a 3}+5 t_{b 3}-7 t_{127}+7 t_{128}\right)$ \\
II & $t_{a 1}+t_{b 1}+t_{a 2}+t_{b 2}+t_{a 3}-t_{b 3}-t_{127}+t_{128}$ & $v_{n \mathrm{~N}}=\frac{V_{\mathrm{DC}}}{2 t_{\mathrm{s}}}\left(t_{a 1}-t_{b 1}-3 t_{a 2}+3 t_{b 2}+5 t_{a 3}-5 t_{b 3}-7 t_{127}+7 t_{128}\right)$ \\
III & $t_{a 1}+t_{b 1}+t_{a 2}-t_{b 2}+t_{a 3}-t_{b 3}-t_{127}+t_{128}$ & $v_{n \mathrm{~N}}=\frac{V_{\mathrm{DC}}}{2 t_{\mathrm{s}}}\left(-t_{a 1}+t_{b 1}+3 t_{a 2}-3 t_{b 2}-5 t_{a 3}+5 t_{b 3}-7 t_{127}+7 t_{128}\right)$ \\
IV & $t_{a 1}-t_{b 1}-t_{a 2}+t_{b 2}+t_{a 3}-t_{b 3}-t_{127}+t_{128}$ & $v_{n \mathrm{~N}}=\frac{V_{\mathrm{DC}}}{2 t_{\mathrm{s}}}\left(t_{a 1}-t_{b 1}-3 t_{a 2}+3 t_{b 2}+5 t_{a 3}-5 t_{b 3}-7 t_{127}+7 t_{128}\right)$ \\
$\mathrm{V}$ & $-t_{a 1}-t_{b 1}+t_{a 2}-t_{b 2}-t_{a 3}+t_{b 3}-t_{127}+t_{128}$ & $v_{n \mathrm{~N}}=\frac{V_{\mathrm{DC}}}{2 t_{\mathrm{s}}}\left(-t_{a 1}+t_{b 1}+3 t_{a 2}-3 t_{b 2}-5 t_{a 3}+5 t_{b 3}-7 t_{127}+7 t_{128}\right)$ \\
VI & $-t_{a 1}-t_{b 1}-t_{a 2}-t_{b 2}+t_{a 3}-t_{b 3}-t_{127}+t_{128}$ & $v_{n \mathrm{~N}}=\frac{V_{\mathrm{DC}}}{2 t_{\mathrm{s}}}\left(t_{a 1}-t_{b 1}-3 t_{a 2}+3 t_{b 2}+5 t_{a 3}-5 t_{b 3}-7 t_{127}+7 t_{128}\right)$ \\
VII & $-t_{a 1}-t_{b 1}-t_{a 2}-t_{b 2}-t_{a 3}-t_{b 3}-t_{127}+t_{128}$ & $v_{n \mathrm{~N}}=\frac{V_{\mathrm{DC}}}{2 t_{\mathrm{s}}}\left(-t_{a 1}+t_{b 1}+3 t_{a 2}-3 t_{b 2}-5 t_{a 3}+5 t_{b 3}-7 t_{127}+7 t_{128}\right)$ \\
\hline \hline
\end{tabular}

TABLE IV

Space Vectors and Line-to-Line Voltages

\begin{tabular}{|c|c|c|}
\hline $\begin{array}{l}\text { Adjacent line-to-line } \\
\text { voltages }\end{array}$ & 1st non-adjacent line-to-line voltages & 2nd non-adjacent line-to-line voltages \\
\hline \multicolumn{3}{|c|}{ Sector I } \\
\hline$V_{12}=\frac{V_{\mathrm{DC}}}{t_{\mathrm{s}}}\left(t_{a 3}\right)$ & $V_{13}=\frac{V_{\mathrm{DC}}}{t_{\mathrm{s}}}\left(t_{a 1}+t_{b 2}+t_{a 1}\right)$ & $V_{14}=\frac{V_{\mathrm{DC}}}{t_{\mathrm{s}}}\left(t_{a 1}+t_{b 1}+t_{a 2}+t_{b 2}+t_{a 3}\right)$ \\
\hline$V_{23}=\frac{V_{\mathrm{DC}}}{t_{\mathrm{s}}}\left(t_{a 1}+t_{b 2}\right)$ & $V_{24}=\frac{V_{\mathrm{DC}}}{t_{\mathrm{s}}}\left(t_{a 1}+t_{b 1}+t_{a 2}+t_{b 2}\right)$ & $V_{25}=\frac{V_{\mathrm{DC}}}{t_{\mathrm{s}}}\left(t_{a 1}+t_{b 1}+t_{a 2}+t_{b 2}+t_{b 3}\right)$ \\
\hline$V_{34}=\frac{V_{\mathrm{DC}}}{t_{\mathrm{s}}}\left(t_{b 1}+t_{a 2}\right)$ & $V_{35}=\frac{V_{\mathrm{DC}}}{t_{\mathrm{s}}}\left(t_{b 1}+t_{a 2}+t_{b 3}\right)$ & $V_{36}=\frac{V_{\mathrm{DC}}}{t_{\mathrm{s}}}\left(t_{b 1}\right)$ \\
\hline$V_{45}=\frac{V_{\mathrm{DC}}}{t_{\mathrm{s}}}\left(t_{b 3}\right)$ & $V_{46}=\frac{V_{\mathrm{DC}}}{t_{\mathrm{s}}}\left(-t_{a 2}\right)$ & $V_{47}=\frac{V_{\mathrm{DC}}}{t_{\mathrm{s}}}\left(-t_{a 1}-t_{b 1}-t_{a 2}\right)$ \\
\hline$V_{56}=\frac{V_{\mathrm{DC}}}{t_{\mathrm{s}}}\left(-t_{a 2}-t_{b 3}\right)$ & $V_{57}=\frac{V_{\mathrm{DC}}}{t_{\mathrm{s}}}\left(-t_{a 1}-t_{b 1}-t_{a 2}-t_{b 3}\right)$ & $V_{51}=\frac{V_{\mathrm{DC}}}{t_{\mathrm{s}}}\left(-t_{a 1}-t_{b 1}-t_{a 2}-t_{b 2}-t_{a 3}-t_{b 3}\right)$ \\
\hline$V_{67}=\frac{V_{\mathrm{DC}}}{t_{\mathrm{s}}}\left(-t_{a 1}-t_{b 1}\right)$ & $V_{61}=\frac{V_{\mathrm{DC}}}{t_{\mathrm{s}}}\left(-t_{a 1}-t_{b 1}-t_{b 2}-t_{a 3}\right)$ & $V_{62}=\frac{V_{\mathrm{DC}}}{t_{\mathrm{s}}}\left(-t_{a 1}-t_{b 1}-t_{b 2}\right)$ \\
\hline$V_{71}=\frac{V_{\mathrm{DC}}}{t_{\mathrm{s}}}\left(-t_{b 2}-t_{a 3}\right)$ & $V_{72}=\frac{V_{\mathrm{DC}}}{t_{\mathrm{s}}}\left(-t_{b 2}\right)$ & $V_{73}=\frac{V_{\mathrm{DC}}}{t_{\mathrm{s}}}\left(t_{a 1}\right)$ \\
\hline \multicolumn{3}{|c|}{ Sector II } \\
\hline$V_{12}=\frac{V_{\mathrm{DC}}}{t_{\mathrm{s}}}\left(-t_{b 3}\right)$ & $V_{13}=\frac{V_{\mathrm{DC}}}{t_{\mathrm{s}}}\left(t_{a 2}\right)$ & $V_{14}=\frac{V_{\mathrm{DC}}}{t_{\mathrm{s}}}\left(t_{a 1}+t_{b 1}+t_{a 2}\right)$ \\
\hline$V_{23}=\frac{V_{\mathrm{DC}}}{t_{\mathrm{s}}}\left(t_{a 2}+t_{b 3}\right)$ & $V_{24}=\frac{V_{\mathrm{DC}}}{t_{\mathrm{s}}}\left(t_{a 1}+t_{b 1}+t_{a 2}+t_{b 3}\right)$ & $V_{25}=\frac{V_{\mathrm{DC}}}{t_{\mathrm{s}}}\left(t_{a 1}+t_{b 1}+t_{a 2}+t_{b 2}+t_{a 3}+t_{b 3}\right)$ \\
\hline$V_{34}=\frac{V_{\mathrm{DC}}}{t_{\mathrm{s}}}\left(t_{a 1}+t_{b 1}\right)$ & $V_{35}=\frac{V_{\mathrm{DC}}}{t_{\mathrm{s}}}\left(t_{a 1}+t_{b 1}+t_{b 2}+t_{a 3}\right)$ & $V_{36}=\frac{V_{\mathrm{DC}}}{t_{\mathrm{s}}}\left(t_{a 1}+t_{b 1}+t_{b 2}\right)$ \\
\hline$V_{45}=\frac{V_{\mathrm{DC}}}{t_{\mathrm{s}}}\left(t_{b 2}+t_{a 3}\right)$ & $V_{46}=\frac{V_{\mathrm{DC}}}{t_{\mathrm{s}}}\left(t_{b 2}\right)$ & $V_{47}=\frac{V_{\mathrm{DC}}}{t_{\mathrm{s}}}\left(-t_{a 1}\right)$ \\
\hline$V_{56}=\frac{V_{\mathrm{DC}}}{t_{\mathrm{s}}}\left(-t_{a 3}\right)$ & $V_{57}=\frac{V_{\mathrm{DC}}}{t_{\mathrm{s}}}\left(-t_{a 1}-t_{b 2}-t_{a 3}\right)$ & $V_{51}=\frac{V_{\mathrm{DC}}}{t_{\mathrm{s}}}\left(-t_{a 1}-t_{b 1}-t_{a 2}-t_{b 2}-t_{a 3}\right)$ \\
\hline$V_{67}=\frac{V_{\mathrm{DC}}}{t_{\mathrm{s}}}\left(-t_{a 1}-t_{b 2}\right)$ & $V_{61}=\frac{V_{\mathrm{DC}}}{t_{\mathrm{s}}}\left(-t_{a 1}-t_{b 1}-t_{a 2}-t_{b 2}\right)$ & $V_{62}=\frac{V_{\mathrm{DC}}}{t_{\mathrm{s}}}\left(-t_{a 1}-t_{b 1}-t_{a 2}-t_{b 2}-t_{b 3}\right)$ \\
\hline$V_{71}=\frac{V_{\mathrm{DC}}}{t_{\mathrm{s}}}\left(-t_{b 1}-t_{a 2}\right)$ & $V_{72}=\frac{V_{\mathrm{DC}}}{t_{\mathrm{s}}}\left(-t_{b 1}-t_{a 2}-t_{b 3}\right)$ & $V_{73}=\frac{V_{\mathrm{DC}}}{t_{\mathrm{s}}}\left(-t_{b 1}\right)$ \\
\hline
\end{tabular}


TABLE V

Space Vector Sectors and Fundamental Signals

\begin{tabular}{cc}
\hline \hline Sector I & Sector II \\
\hline$V_{1}>V_{2}>V_{7}>V_{3}>V_{6}>V_{4}>V_{5}$ & $V_{7}>V_{1}>V_{3}>V_{7}>V_{4}>V_{6}>V_{5}$ \\
\hline Sector III & Sector IV \\
$V_{2}>V_{3}>V_{1}>V_{4}>V_{7}>V_{5}>V_{6}$ & $V_{3}>V_{2}>V_{4}>V_{1}>V_{5}>V_{7}>V_{6}$ \\
\hline Sector V & Sector VI \\
$V_{3}>V_{4}>V_{2}>V_{5}>V_{1}>V_{6}>V_{7}$ & $V_{4}>V_{3}>V_{5}>V_{2}>V_{6}>V_{1}>V_{7}$ \\
\hline Sector VII & Sector VIII \\
$V_{4}>V_{5}>V_{3}>V_{6}>V_{2}>V_{7}>V_{1}$ & $V_{5}>V_{4}>V_{6}>V_{3}>V_{7}>V_{2}>V_{1}$ \\
\hline Sector IX & Sector X \\
$V_{5}>V_{6}>V_{4}>V_{7}>V_{3}>V_{1}>V_{2}$ & $V_{6}>V_{5}>V_{7}>V_{4}>V_{1}>V_{3}>V_{2}$ \\
\hline Sector $\mathbf{X I}$ & Sector $\mathbf{X I I}$ \\
$V_{6}>V_{7}>V_{5}>V_{1}>V_{4}>V_{2}>V_{3}$ & $V_{7}>V_{6}>V_{1}>V_{5}>V_{2}>V_{4}>V_{3}$ \\
\hline Sector XIII & Sector XIV \\
$V_{7}>V_{1}>V_{6}>V_{2}>V_{5}>V_{3}>V_{4}$ & $V_{1}>V_{7}>V_{2}>V_{6}>V_{3}>V_{5}>V_{4}$ \\
\hline \hline
\end{tabular}

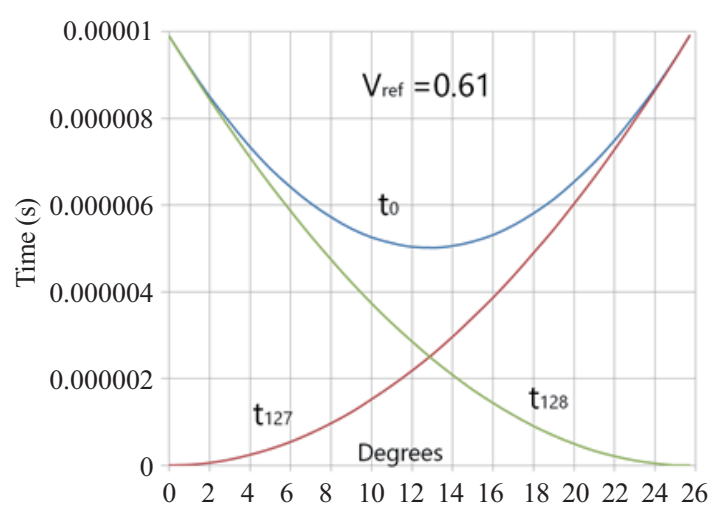

Fig. 7. Application time of zero vector in sector I.

relationship exists for the sectors from VIII to XIV but with opposite polarity to that of sectors I to VII, respectively. A direct relationship between line-to-line voltages and space vectors is thus evident from Table IV. Further, it can be noted that the line-to-line voltages are determined by only active vectors. Hence line-to-line output voltage can be obtained directly using the time of application.

\section{E. Space Vector Sectors and Fundamental Signals}

The space vectors and the fundamental signals are shown in Fig. 6 and their relationship is listed in Table V. The sectors are shown in Roman letter I to XIV and the fundamental signals are seven-phase sinusoidal waveform.

\section{F. Distribution of Zero-Sequence Signal and Zero Vectors}

In SVPWM the two zero vectors timings $t_{127}, t_{128}$ can be distributed as per choice depending upon the type of PWM to be used. That means the timings are flexible in SVPWM whereas timings are fixed in CBPWM. Depending on the choice of $t_{127}$ and $t_{128}$ various space vector PWM are possible. The expression for $t_{127}(0000000)$ can be written as (15)-(17). Fig. 7 shows the variation of zero vector application time in

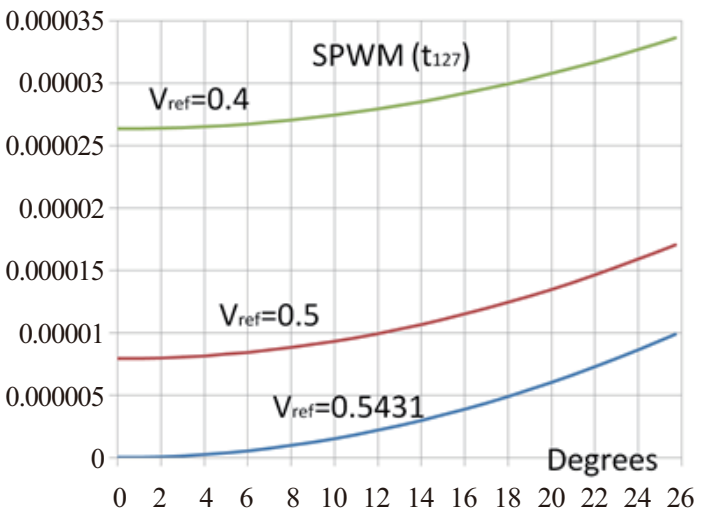

Fig. 8. Variation of $t_{127}$ with angle in sector I for sinusoidal Carrier-based PWM.

sector I for maximum possible reference of 0.61 p.u. The switching frequency is assumed as $5 \mathrm{kHz}$. The general form of distribution of $t_{127}$ and $t_{128}$ can be written as:

$$
\begin{gathered}
t_{127}=\xi(t) t_{o} \\
t_{128}=[1-\xi(t)] t_{o} \\
t_{127}+t_{128}=t_{\mathrm{s}}-\left(t_{a 1}+t_{b 1}+t_{a 2}+t_{b 2}+t_{a 3}+t_{b 3}\right)
\end{gathered}
$$

where, $0 \leqslant \xi(t) \leqslant 1$ A degree of freedom that exists in CBPWM is the shape of zero-sequence signal. In general, the zerosequence signal can be expressed as [23]:

$$
v_{n \mathrm{~N}}=\xi(t)\left(1-V_{\max }\right)+[1-\xi(t)]\left(-1-V_{\min }\right)
$$

By using (18) for arbitrary zero-sequence signal, the timings of application of zero space vectors $t_{127}$ and $t_{128}$ can be obtained.

\section{1) Sinusoidal Pulse Width Modulation (SPWM)}

This is the result of $v_{n \mathrm{~N}}(t)=0.0$ which leads to SPWM with modulation index $M_{\mathrm{SPWM}}=1$. The equivalent distribution of $t_{127}$ and $t_{128}$ is obtained using (17). Considering switching period of $0.2 \mathrm{~ms}$, the variation of application time of in sector I without harmonic injection is shown in Fig. 8.

$$
\begin{aligned}
& t_{127}=\frac{t_{\mathrm{s}}}{2}\left[1-\frac{1}{2}\left(\frac{6 k_{1}+4 k_{2}+2}{k_{3}}\right)\left|v_{\mathrm{s}}^{*}\right| \cos (\theta)\right] \\
& t_{128}=t_{\mathrm{s}}-\left(t_{a 1}+t_{b 1}+t_{a 2}+t_{b 2}+t_{a 3}+t_{b 3}+t_{127}\right)
\end{aligned}
$$

\section{2) Seventh Harmonic Injection PWM (SHIPWM)}

This is the result of $v_{n \mathrm{~N}}=V_{1} \frac{\sin (\pi / 14)}{7}$ which leads to SHIPWM with maximum modulation index $M_{\text {SHIPWM }}=1.0318$. The corresponding distribution of $t_{127}$ and $t_{128}$ is

$t_{127}=\frac{t_{\mathrm{s}}}{2}\left\{1-\frac{1}{2}\left(\frac{6 k_{1}+4 k_{4}+2}{k_{3}}\right)\left|v_{\mathrm{s}}{ }^{*}\right|\left[\cos (\theta)+\frac{\sin \left(\frac{\pi}{14}\right)}{7} \cos (7 \theta)\right]\right\}$ 


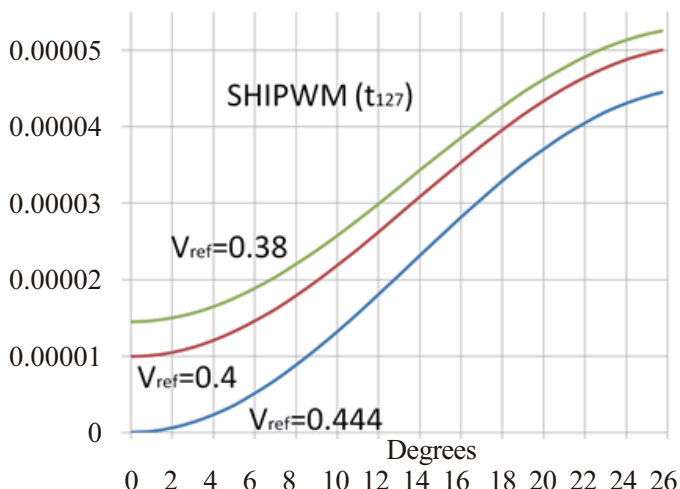

Fig. 9. In sector I, the variation of $t_{127}$ with angle for SHIPWM.

$$
t_{128}=t_{\mathrm{s}}-\left(t_{a 1}+t_{b 1}+t_{a 2}+t_{b 2}+t_{a 3}+t_{b 3}+t_{127}\right)
$$

Fig. 9 shows, in sector I, the variation of application time of $t_{127}$ with harmonic injection.

\section{3) Offset Addition or Triangular Zero-Sequence Injection PWM (TIPWM)}

This is the result of $v_{n \mathrm{~N}}(t)=-\frac{1}{2}\left(V_{\max }+V_{\min }\right)$ which leads to TIPWM with maximum modulation index 1.0318. This is due to the application of zero vectors. The corresponding distribution of $t_{127}$ and $t_{128}$ is

$$
t_{127}=t_{128}=\frac{1}{2}\left[t_{\mathrm{s}}-\left(t_{a 1}+t_{b 1}+t_{a 2}+t_{b 2}+t_{a 3}+t_{b 3}\right)\right]
$$

\section{Switching Pattern of CARrier-Based PWM AND Space Vector PWM}

Carrier-based PWM is characterized by the type of high carrier signals that are used to compare with low frequency modulating signals. Performance of the modulation scheme heavily relies on the shape of carrier signals. Usually triangular (double edge) and saw-tooth (single edge) waveforms are used as carrier signals. In contrast space vector method optimally determines the active and zero vector pulse width to satisfy the volt-second balance. The proper sequencing of the active and zero vectors determine the performance of space vector modulator. For triangular carrier signal, the equivalent switching pattern corresponds to the one used in space vector PWM (Fig. 5). Thus the two modulation schemes yield similar performance. Considering saw-tooth waveform as the carrier signal the equivalent switching pattern is illustrated in Fig. 10.

\section{EXPERIMENTAL RESULTS}

A seven-phase VSI is built using fourteen insulated gate bipolar transistor (IGBT) power modules. The gate pulses were generated from the Matlab/Simulink model, using dSPACE DS1103 control board. A star-connected seven-phase $R-L$ load $(R=75$ $\Omega, L=78 \mathrm{mH}$ ) is connected to each output phase of the sevenphase inverter. DC link voltage is adjusted to $V_{\mathrm{DC}}=200 \mathrm{~V}$ for all PWM schemes from a regulated DC power supply. The

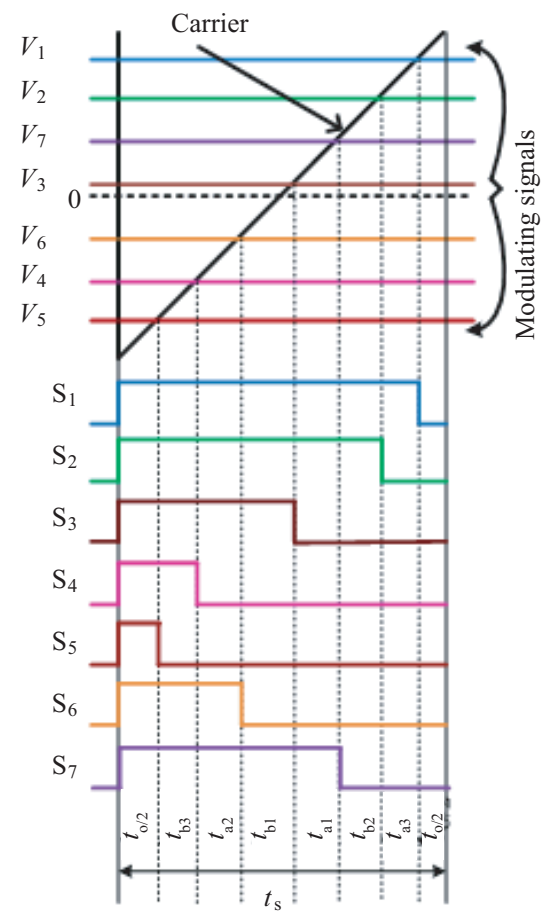

Fig. 10. Switching pattern for carrier based PWM with saw tooth carrier.

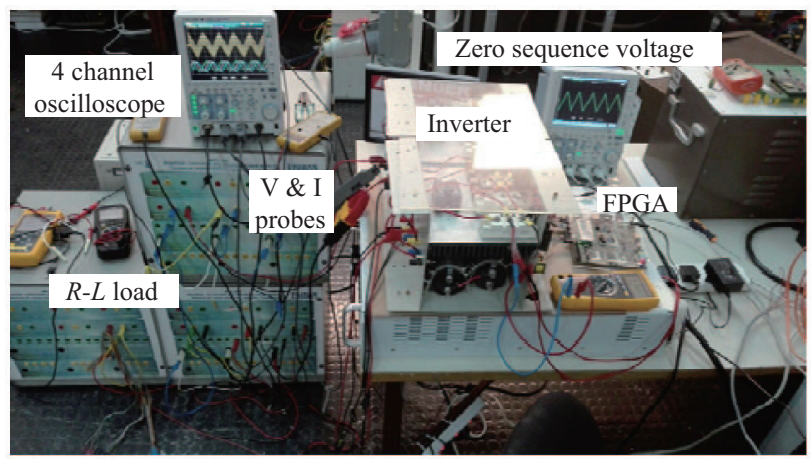

Fig. 11. Experimental setup.

dead time between switching on the lower leg switch and switching off the upper leg switch is kept at $2 \mu$ s by dead time code in the FPGA Vertex-5 board (connected externally to the dSpace board DS 5203) which is used in conjunction with dSpace-1006. The minimum sampling time used is $10 \mu$ s for dspace and $100 \mathrm{~ns}$ for the FPGA. The experimental set-up is shown in Fig. 11, where, experiments were performed with CBPWM, SHIPWM, SVPWM, and TIPWM and results are shown in Figs. 12-14.

DC link voltage is adjusted to $V_{\mathrm{DC}}=200 \mathrm{~V}$ from a regulated DC power supply for controlling the load with voltage/ frequency $=$ constant. Fig. 12 shows experimental results of four PWM schemes with a unity modulation index, where from the top, 1st trace gives the phase-A voltage, 2nd trace gives the A-B line voltage, 3rd trace gives the A-C line to voltage and the 4 th trace gives the phase-A current. The current is shown for phase-A, but other phase currents will have same magnitude but phase shifted by $\frac{2 \pi}{7}$ degrees. Fig. 12(a), (b), (c) 


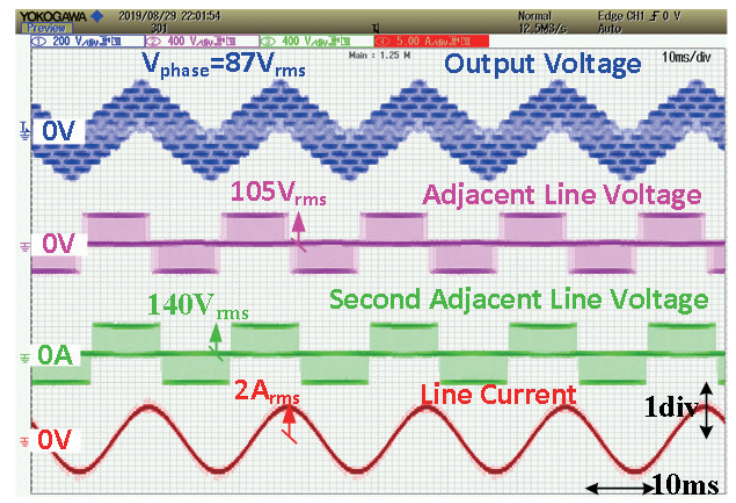

(a)

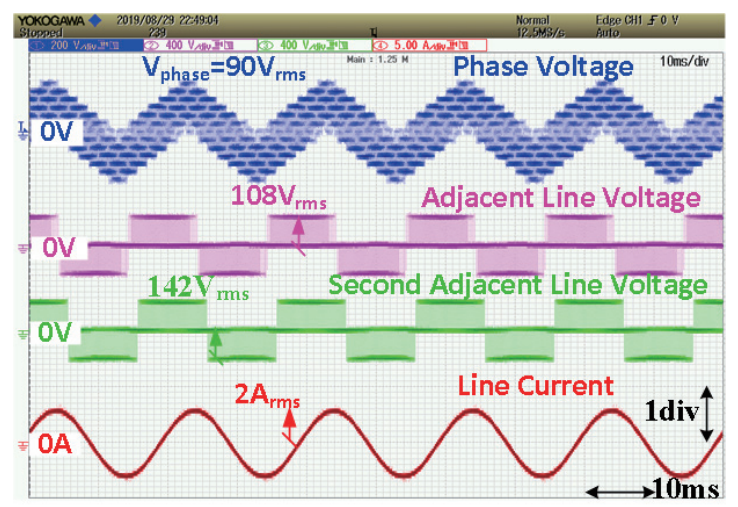

(c)

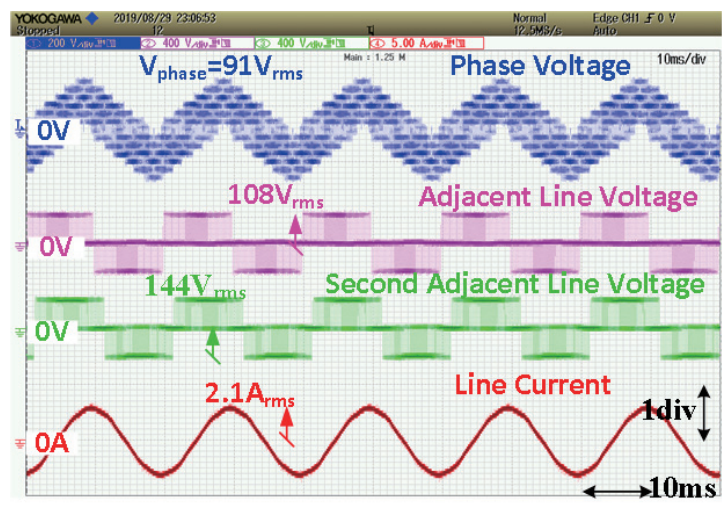

(b)

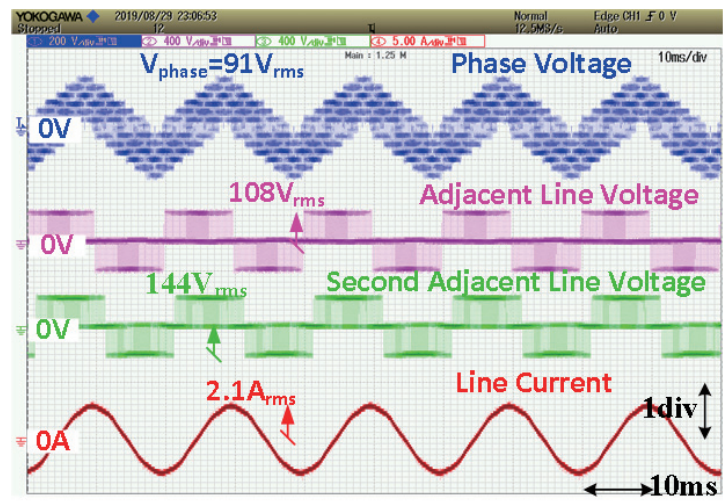

(d)

Fig. 12. With modulatling Index $m=1$, phase voltage, adjacent line voltage $\left(V_{\mathrm{AB}}\right)$, second adjacent voltage $\left(V_{\mathrm{AC}}\right)$, and phase current. (a) CBPWM. (b)SVPWM. (c) SHIPWM. (d) TIPWM.

and (d) show the experimental results for CBPWM, SHIPWM, SVPWM, and TIPWM, respectively. The output voltage at a modulation index of unity is presented in Fig. 12; the RMS of phase voltage is seen as $87 \mathrm{~V}$ for the CBPWM while the theoretical value is $85.2 \mathrm{~V}$ that amounts to an error of $3.7 \%$. This difference appears due to dead time in the experimental realization and higher sampling rate.

In this paper, due to the laboratory constraints, the experiment has been done on a 7-phase $R-L$ load instead of 7-phase induction motor. However, all PWM techniques have been validated for different modulation index values with constant voltage/frequency $=$ constant control. The experimental results for the under modulation (M.I = 0.4) and over modulation (M.I $=1.2$ ) for all four PWM techniques are presented in Fig. 13, where the phase-A voltage, A-B line to line voltage, $\mathrm{A}-\mathrm{C}$ line to line voltage and phase-A current are shown. From this figure it is observed that because of the $V / f=$ constant control the lineline voltage and frequency is maintained constant for 0.4 and 1.2 M.I values. With CBPWM, the RMS line to A-B line voltage and frequency of the load are $42 \mathrm{~V}$ and $20 \mathrm{~Hz}$, respectively for 0.4 M.I, as shown in Fig. 13(a). Similarly, A-B line voltage and frequency of the load are $120 \mathrm{~V}$ and $60 \mathrm{~Hz}$ respectively for M.I = 1.2, as shown in Fig. 13(b). The set of results for 0.4 and 1.2 M.I values with SHIPWM, SVPWM, and TIPWM are shown in Fig.13(c) to (f), respectively. Since the SVPWM and TIPWM will generate same modulating waveform, so the phase voltage and the currents will be the ame. Because of this, the results are shown for SVPWM in Fig. 13(c) and (d).

The experimental phase voltage, current waveforms and associated harmonic spectrum with CBPWM technique for different modulation index values are presented in Fig. 14. The percentage THD values of phase voltage and phase current for M.I = 1 with CBPWM are shown in Fig. 14(b), where it is observed that the current and voltage THD is $1.8 \%$ and $7.2 \%$, respectively. However, the percentage THDs for remaining carrier-based PWMs are clearly mentioned in Table VI. Outputs of all carrier-based PWMs are similar with slight difference. This difference is due to the modulation index is in over-modulation region.

\section{CONCLUSION}

The sinusoidal output is achieved using CBPWM and SVPWM schemes, both are analyzed/compared using analytic, simulation and experimental approaches. A transformation between arbitrary CBPWM and SVPWM is shown by relating space vectors and modulation signals. The line-to-line voltage and space vectors are shown to be related directly. It establishes that only active vectors give the line-to-line voltage. The line- 


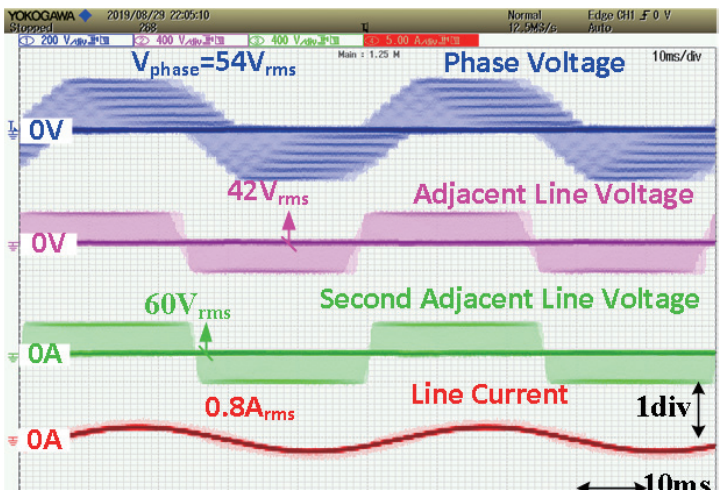

(a)

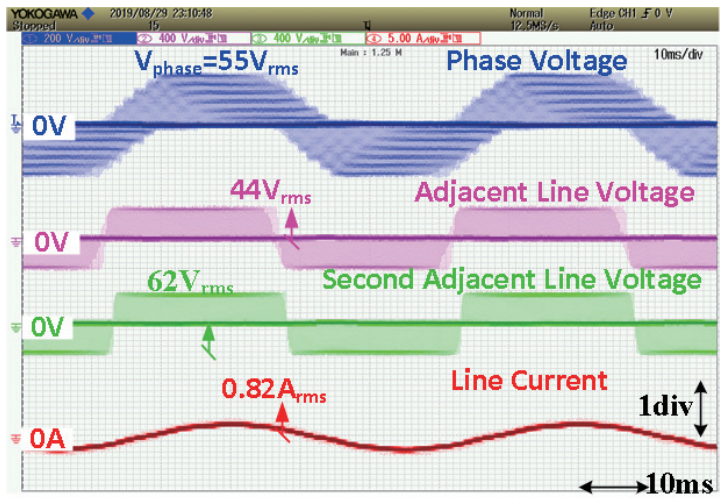

(c)

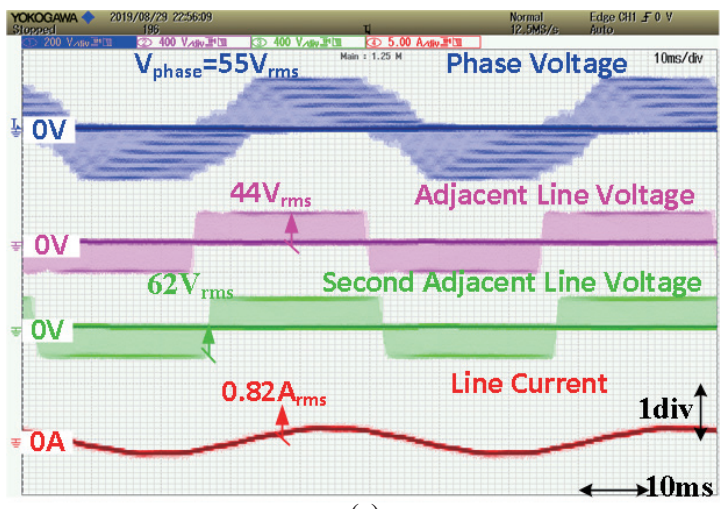

(e)

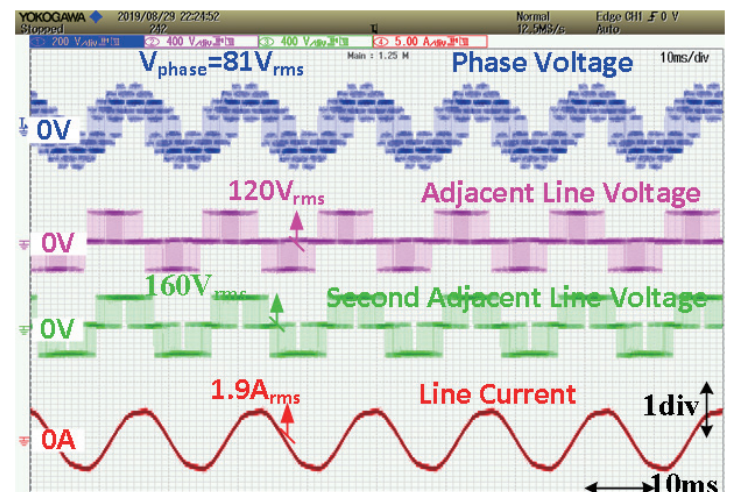

(b)

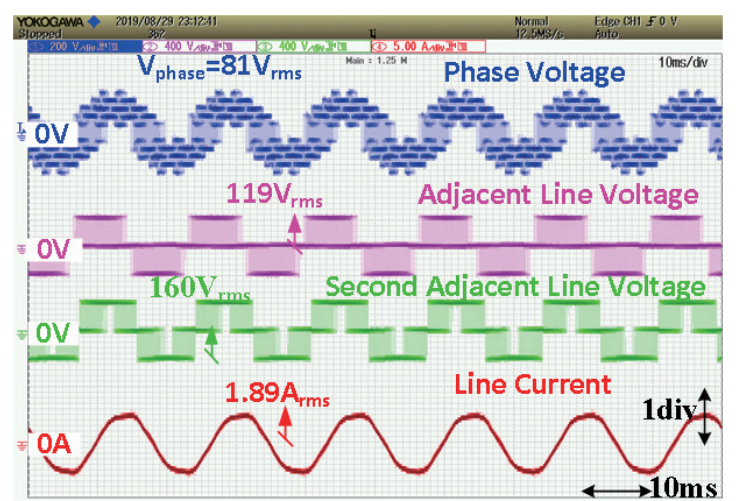

(d)

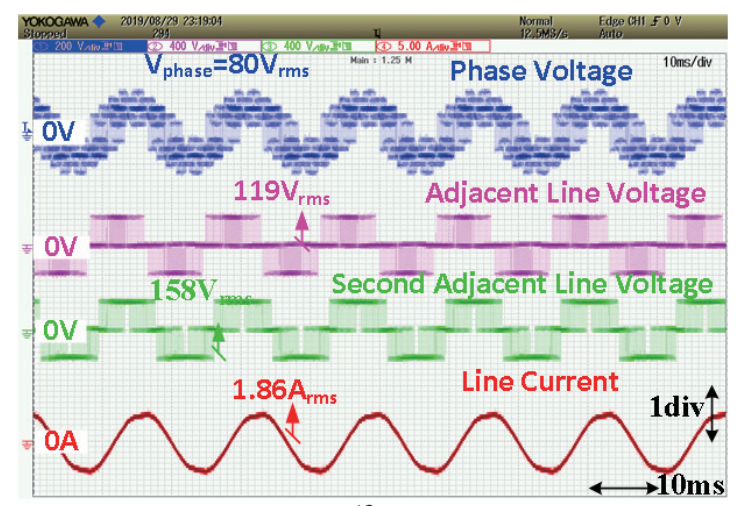

(f)

Fig. 13. Phase voltage, adjacent line voltage $\left(V_{\mathrm{AB}}\right)$, second adjacent voltage $\left(V_{\mathrm{AC}}\right)$, and phase current. (a) At $m=0.4$, CBPWM. (b) At $m=1.2$, CBPWM (over modulation). (c)At $m=0.4$, SVPWM (under modulation). (d) At $m=1.2$, SVPWM (over modulation). (e) At $m=0.4$, SHIPWM (under modulation). (f) At $m$ $=1.2$, SHIPWM (under modulation).

TABLE VI

COMPARISON OF EXPERIMENTAL Results

\begin{tabular}{cccccc}
\hline \hline S1. No. & PWM type & Mod. index & $V_{\text {phase }}(\mathrm{V})$ & $I_{\text {phase }}(\mathrm{A})$ & $\begin{array}{c}\text { Current THD } \\
(\%)\end{array}$ \\
\hline 1 & SVPWM & $V_{\text {ref }}=1.0318$ & 91 & 2.1 & 1.4 \\
3 & CBPWM & 1.0 & 87 & 2 & 2.8 \\
5 & SHIPWM & 1.0318 & 90 & 2.1 & 2.2 \\
7 & TIPWM & 1.0318 & 91 & 2.1 & 1.4 \\
\hline \hline
\end{tabular}

to-line voltage is independent of zero vectors. The space vector in various sectors and modulation signals are related. The distribution of zero vectors application times for SPWM,
SHIPWM and TIPWM are expressed analytically. In terms of THD as performance modulator for triangular and saw-tooth carrier signals are shown. Finally, it is concluded that during implementation similarities do exist between space vector PWM and carrier-based PWM, and shown to have underlying relationships in a seven-phase VSI similar to three-phase VSI. With the increase of phase numbers the space vector PWM method becomes more and more complex and the gain the output voltage reduces. It is further concluded that the third harmonic injection and triangular injection methods give similar results as that of SVPWM, however, they are simple to implement. Hence for the practical purposes, instead of using SVPWM, triangular injection and 3rd harmonic injection method should be preferred. 


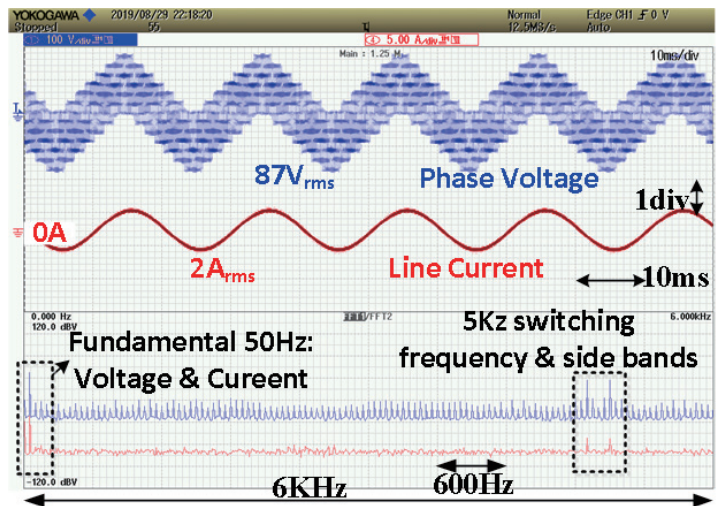

(a)

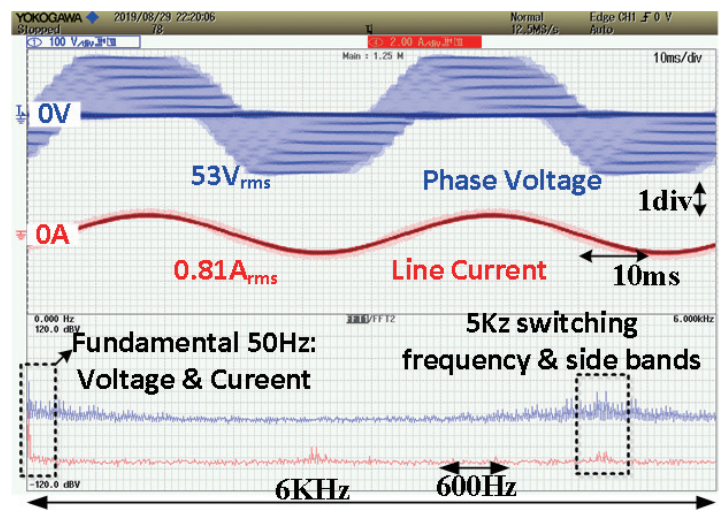

(c)

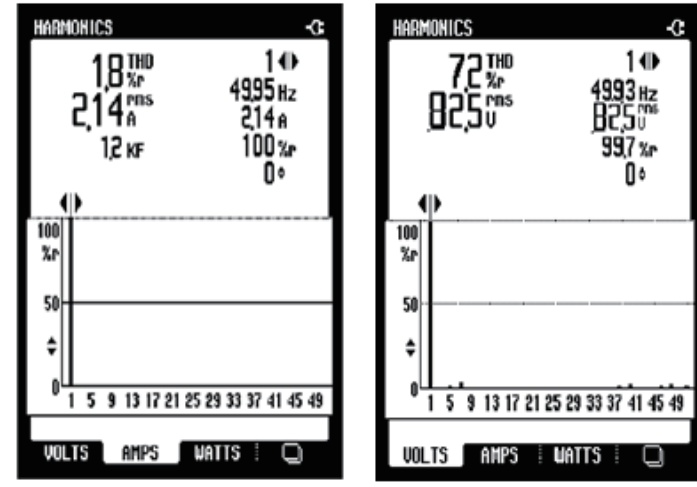

(b)

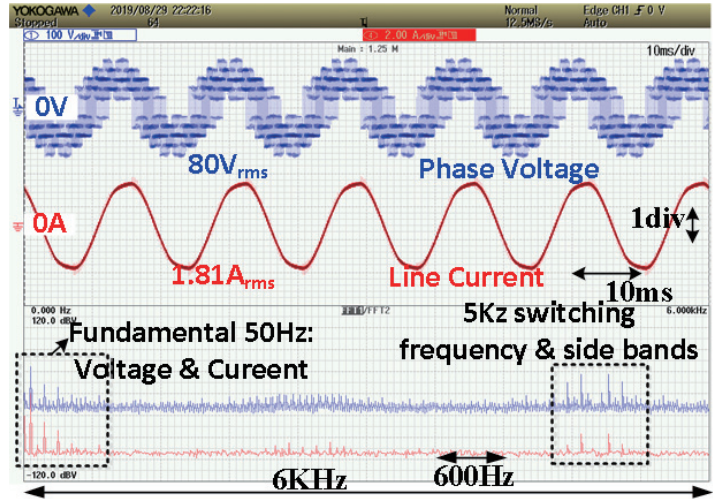

(d)

Fig. 14. Harmonic analysis of the CBPWM at different modulation indices and in $V / f$ control mode. (a) At $50 \mathrm{~Hz}$ operation. (b) Lower order harmonics. (c) At 20 Hz operation. (d) At $60 \mathrm{~Hz}$ operation.

\section{REFERENCES}

[1] M. Jones and E. Levi, "A literature survey of state-of-the-art in multiphase AC drives," Proc. 36th Universities Power Engineering Conference UPEC, Stafford, UK, 2002, pp. 505-510.

[2] E. Levi, R. Bojoi, F. Profumo, H. Toliyat, and S. Williamson, "Multiphase induction motor drives - a technology status review," IEEE Transactions on Industrial Electronics, vol. 1, no. 4, pp. 489-516, 2007.

[3] E. Levi, "Multiphase electric machines for variable-speed applications," IEEE Transactions on Industrial Electronics, vol. 55, no. 5, pp. 1893 1909, May 2008.

[4] E. Levi, "Advances in converter control and innovative exploitation of additional degrees of freedom for multiphase machines," IEEE Transactions on Industrial Electronics, vol. 63, no. 1, pp. 433-448, Jan. 2016.

[5] F. Barrero and M. J. Duran, "Recent advances in the design, modeling and control of multiphase machines-Part 1," IEEE Transac-tions on Idustrial Electronics, vol. 63, no. 1, pp. 449-458, Jan. 2016.

[6] M. J. Duran and F. Barrero, "Recent advances in the design, modeling and control of multiphase machines-Part 2," IEEE Transactions on Industrial Electronics, vol. 63, no. 1, pp. 459-468, Jan. 2016.

[7] H. Abu-Rub, A. Iqbal, and J. Guzinski, High Performance AC Drives with Matalb/Simulink Models. UK. Wiley Press, Apr. 2012.

[8] Fahem, K, Chariag, DE, Sbita, L, "Performance evaluation of continuous and discontinuous pulse width modulation techniques for grid connected PWM converter," International Transactions on Electrical Energy Systems. 28(10), 2018; 28:e2609.

[9] A. Iqbal and E. Levi, "Space vector modulation scheme for a five-phase voltage source inverter," Proc. European Power Electronics and Appl. Conf., EPE, Dresden, Germany, 2005, DOI: 10.1109/EPE.2005.219194

[10] A. Iqbal and E. Levi, "Space vector PWM techniques for sinusoidal output voltage generation with a five-phase voltage source inverter," Electric Power Components and Systems, vol. 34, no. 2, 2006, pp. 119140.

[11] K. Y. Chen and Y. L. Xie, "Reducing harmonics distortion in five-phase VSI using space-vector-based optimal hybrid PWM," IEEE Transactions on Power Electronics, vol. 32, no. 3, pp. 2098-2113, March 2017.

[12] M. Priestley, J. E. Fletcher, and C. Tan, "Space-vector PWM technique for five-phase open-end winding PMSM drive operating in the overmodulation region," in IEEE Transactions on Industrial Electronics, vol. 65, no. 9, pp. 6816-6827, Sept. 2018.

[13] E. A. R. Engku Ariff, O. Dordevic and M. Jones, "A space vector PWM technique for a three-level symmetrical six-phase drive," in IEEE Transactions on Industrial Electronics, vol. 64, no. 11, pp. 8396-8405, Nov. 2017.

[14] M. S. A. Shaikh and R. Maurya, "Realization of 24-sector SVPWM with new switching pattern for six-phase induction motor drive," IEEE Transactions on Power Electronics. vol. 34, no. 6, pp. 1-1. 2018. DOI: 10.1109/TPEL.2018.2870952.

[15] C. Wang, K. Wang, and X. You, "Research on synchronized SVPWM strategies under low switching frequency for six-phase VSI-fed asymmetrical dual stator induction machine," IEEE Transactions on Industrial Electronics, vol. 63, no. 11, pp. 6767-6776, Nov. 2016.

[16] K.A. Chinmaya and G.K. Singh, "Experimental analysis of various spacevector pulse width modulation (SVPWM) techniques for dual three. phase induction motor drive," International Transactions on Electrical Energy Systems, 29(1), 2019, p.e2678.

[17] G. Grandi, G. Serra, and A. Tani, "Space vector modulation of a sevenphase voltage source inverter," Proc. Int. Symp. Power Electronics, Electrical Drives Automation and Motion SPEEDAM, Taormina, Italy, 2006, pp. 1149-1156.

[18] O. Dordevic, E. Levi, and M. Jones, "A vector space decomposition 
based space vector PWM algorithm for a three-level seven-phase voltage source inverter," IEEE Transactions on Power Electronics, vol. 28, no. 2, pp. 637-649, Feb. 2013.

[19] B. P. Reddy and S. Keerthipati, "A multilevel inverter configuration for an open-end-winding pole-phase-modulated-multiphase induction motor drive using dual inverter principle," in IEEE Transactions on Industrial Electronics, vol. 65, no. 4, pp. 3035-3044, April 2018.

[20] B. P. Reddy, M. Rao A, M. Sahoo, and S. Keerthipati, "A fault-tolerant multilevel inverter for improving the performance of a pole-phase modulated nine-phase induction motor drive," in IEEE Transactions on Industrial Electronics, vol. 65, no. 2, pp. 1107-1116, Feb. 2018.

[21] S. Moinoddin, H. Abu-Rub, A. Iqbal, Sk. M. Ahmed, O. Dordevic, and E. Levi, "Space vector pulse-width modulation technique for an elevenphase voltage source inverter with sinusoidal output voltage generation," IET Power Electronics, 2015, vol. 8, no. 6, pp. 1000-1008.

[22] S. Moinoddin, H. Abu-Rub, and A. Iqbal, "Modelling and implementation of SVPWM technique for a thirteen-phase voltage source inverter-sinusoidal output waveform", IEEE Applied Power Electronics Conference and Exposition (APEC) 2014, Fort Worth, Texas, pp. 268273 March 16-20, 2014.

[23] S. Keerthipati and P. R. B, "Linear modulation range and torque ripple profile improvement of PPMIM drives," in IEEE Transactions on Power Electronics. 2019, DOI: 10.1109/TPEL.2019.2907782

[24] S. Moinoddin, H. Abu-Rub, A. Iqbal, and R. Alammari, "Modelling and implementation of SVPWM technique for a fifteen-phase voltage source inverter for sinusoidal output," IEEE Int. conf. on Industrial Technology ICIT-2015, 16-19 March, Seville, Spain, pp. 1075-1080.

[25] Ahoora Bahram and Mehdi Narimani, "A sinusoidal pulsewidth modulation (SPWM) technique for capacitor voltage balancing of a nested t-type four-level inverter," IEEE Transactions on Power Electronics, vol. 34, no. 2, pp. 1008-1012 2, 2019.

[26] S. Karugaba and O. Ojo, "A carrier-based PWM modulation technique for balanced and unbalanced reference voltages in multiphase voltagesource inverters," IEEE Transactions on Industry Applications, vol. 48, no. 6, pp. 2102-2109, Nov.-Dec. 2012.

[27] A. Iqbal, E. Levi, M. Jones, and S.N. Vukosavic, "Generalised sinusoidal PWM with harmonic injection for multi-phase VSIs," Proc. IEEE Power Elect. Spec. Conf., PESC, Jeju, Korea, 2006, pp. 2871-2877. DOI:10.1109/pesc.2006.1712206

[28] G.D. Holmes and T.A. Lipo, "Pulse width modulation for power converters-principle and practice," IEEE Press-Series on Power Engineering, John Wiley and Sons, Piscataway, NJ, USA, 2003.

[29] Y. Liu, X. Ge, X. Feng, and R. Ding, "Relationship between SVPWM andcarrier-based PWM of eight-switch three-phase inverter," Electronics Letters, vol. 51, no. 13, pp. 1018-1019, 25. 6. 2015.

[30] V. Blasko, "Analysis of a hybrid PWM based on modified space vectorand triangle comparison method," IEEE Transactions on Industry Applications, vol. 33, pp.756-764, May/June 1997.

[31] Keliang Zhou and Danwei Wang, "Relationship between space-vector modulation and three-phase carrier-based PWM: a comprehensive analysis [three-phase inverters]," in IEEE Transactions on Industrial Electronics, vol. 49, no. 1, pp. 186-196, Feb. 2002.

[32] H. Fang, X. Feng, W. Song, X. Ge, and R. Ding, "Relationship betweentwo-level space-vector pulse-width modulation and carrierbased pulse width modulation in the over-modulation region," IET Power Electronics, vol. 7, no. 1, pp. 189-199, January 2014.

[33] A. Iqbal and S. Moinuddin, "Comprehensive relationship between carrier-based PWM and space vector PWM in a five-phase VSI," IEEE Transactions on Power Electronics, vol. 24, no. 10, 2009, DOI: 10.1109/ TPEL.2009.2029051

[34] A. Iqbal, S. Moinuddin, S. Ahmed, M. Ali, A. Sarwar, and K. Munde, Power Electronics Handbook (4e), Elsevier, Chapter 15, Multiphase Converter, pp. 457-528, 2018.

[35] A. A. Abduallah, M. Meraj, M. Al-Hitmi, and A. Iqbal, "Space vector pulse width modulation control techniques for a five-phase quasiimpedance source inverter," in IET Electric Power Applications, vol. 12, no. 3, pp. 379-387, Mar. 2018.
[36] C. Tan, D. Xiao, John Edward Fletcher, and M. Fazlur Rahman, "Analytical and experimental comparison of carrier-based PWM methods for the five-phase coupled-inductor inverter," IEEE Transactions on Industrial Electronics, vol. 63, no. 12, 2016, pp. 73287338.

[37] Bo Tan, Zhuobin Gu, Ke Shen, and Xiaofeng Ding, "Third harmonic injection SPWM method based on alternating carrier polarity to suppress the common mode voltage," IEEE Access, vol. 7, 2019, pp. 9805-9816, DOI:10.1109/ACCESS.2018.2890014

[38] B. P. Reddy and S. Keerthipati, "Torque ripple minimization of PPMIM drives with phase-shifted carrier PWM," IECON 2018-44th Annual Conference of the IEEE Industrial Electronics Society, Washington, DC, 2018, pp. 725-730.

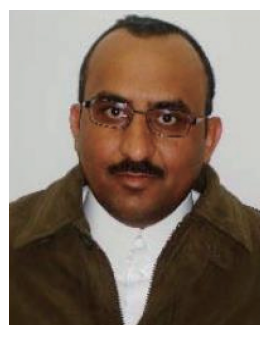

Mohammed A. Al-Hitmi was born in Qatar, in 1968. He received his B.Sc. degree in Electrical Engineering from Qatar University, Doha, Qatar, in 1992, and his M.Sc. and Ph.D. degrees in Control Engineering from the University of Sheffield, Sheffield, ENG, UK, in 1994 and 2002, respectively. He is presently working as an Assistant Professor in the Department of Electrical Engineering, Qatar University. His current research interests include control systems theory, neural networks, fuzzy control and electric drive systems.

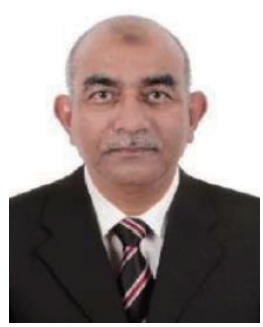

Shaikh Moinoddin, Ph.D., Former Assistant Professor at Electrical Engineering, Aligarh Muslim University (AMU), Aligarh, India. He received his B.E. (Two Gold Medals), M.Sc. Engineering (Power System \& Drives) degrees and Ph.D. in 1996, 1999, and 2009 respectively, from the Aligarh Muslim University (AMU), Aligarh, India. He served as Airman (Sergeant) in Indian Air Force from 1972 to 1987 (16 years). He had been employed as Foreman (Electrical) and then as Lecturer in Electrical Engineering Section, University Polytechnic, AMU, Aligarh from 2001 to 2019 where he served as Assistant Professor until February 2019 (retired on superannuation). He served as an Assistant Professor and Assistant Research Scientist in Sebha University, Libya and Texas A \& M University at Qatar from 2009 to 2011 and 2011 to 2014 respectively.

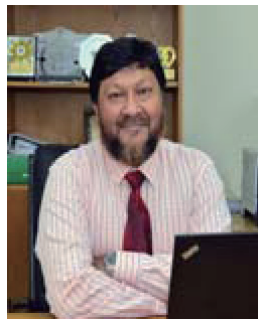

Atif Iqbal received B.Sc. (Gold Medal) and M.Sc. Engineering (Power System\&Drives) degrees in 1991 and 1996, respectively, from the Aligarh Muslim University (AMU), Aligarh, India, and Ph.D. in 2006 from Liverpool John Moores University, Liverpool, UK. He became Fellow IET (UK) in 2018, Fellow IE (India) in 2012 and Senior Member IEEE in 2011. Currently, he is an Associate Professor at Electrical Engineering, Qatar University, Qatar. He has been employed as a Lecturer in the Department of Electrical Engineering, AMU, Aligarh since 1991 where he served as Full Professor until Aug. 2016. He has received the Best Research Papers Awards at IEEE ICIT-2013, IET-SESICON-2013, and SIGMA 2018. He has authored/co-authored more than 300 research papers and one book and three books chapters. His principal area of research interest is Power Electronic, multi-phase motor drives and Renewable Energy sources. 


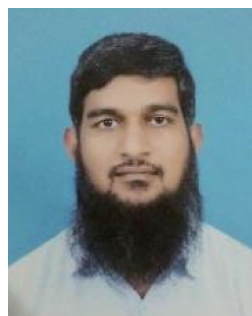

Khaliqur Rahman was born in Uttar Pradesh, India, in 1986. He received his B.Tech. and M.Tech. degrees from Aligarh Muslim University (AMU), Aligarh, India, in 2008 and 2010, respectively. From July 2010 to June 2012, he was a Lecturer in the Department of Electrical Engineering, Aligarh Muslim University. He received his Ph.D. degree from the Qatar University, Doha, Qatar in 2019.

$\mathrm{He}$ is presently working as an Assistant Professor in Aligarh Muslim University. His current research interests include the modeling and control of multiphase power electronic converters.

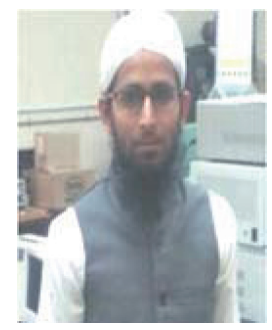

Mohammad Meraj received the bachelor's in Electrical Engineering from the Osmania University, Hyderabad, India and and Master's degree Machine Drives and Power Electronics from IIT Kharagpur, India, in 2012 and 2014, respectively. Currently, he is working towards a Ph.D. degree in Electrical Engineering from the Qatar University, Qatar. His interest includes power electronics, DC/AC converter, DC-DC converter, and renewable energy. 